\title{
Nonlinear equilibration of a dynamo in a smooth helical flow
}

\author{
By ANDREW P. BASSOM AND ANDREW D. GILBERT \\ Department of Mathematics, University of Exeter, North Park Road, Exeter, \\ Devon, EX4 4QE, UK
}

(Received 7 August 1996 and in revised form 8 January 1997)

We investigate the nonlinear equilibration of magnetic fields in a smooth helical flow at large Reynolds number $R e$ and magnetic Reynolds number $R m$ with $R e \gg R m \gg 1$. We start with a smooth spiral Couette flow driven by boundary conditions. Such flows act as dynamos, that is are unstable to growing magnetic fields; here we disregard purely hydrodynamic instabilities such as Taylor-Couette modes. The dominant feedback from a magnetic field mode is only on the mean flow and this yields a simplified 'mean-flow system' consisting of one magnetic mode and the mean flow, which we solve numerically. We also obtain the asymptotic structure of the equilibrated fields for weakly and strongly nonlinear regimes. In particular the field tends to concentrate in a cylindrical shell where all stretching and differential rotation is suppressed by the Lorentz force, and the fluid is in solid-body motion. This shell is bounded by thin diffusive layers where the stretching that maintains the field against diffusive decay is dominant.

\section{Introduction}

In this paper we discuss the nonlinear evolution and equilibration of growing magnetic fields in a family of dynamos first investigated by Ponomarenko (1973). These are axisymmetric helical flows taking the general form

$$
\boldsymbol{u}=(0, v(r), w(r)), \quad v(r) \equiv r \omega(r),
$$

in cylindrical polar coordinates $(r, \theta, z)$. Provided the fluid conducts electrical currents sufficiently well, many flows of this type are kinematic dynamos and amplify seed magnetic fields, which may be taken of the form

$$
\boldsymbol{b}=(a(r), b(r), c(r)) \exp (\mathrm{i} m \theta+\mathrm{i} k z+\mathrm{i} \varpi t)+\text { c.c. } \quad(m>0, k \neq 0),
$$

where 'c.c.' denotes complex conjugate. These Ponomarenko dynamos have been discussed as models of magnetic field generation in galactic jets (Shukurov \& Sokoloff 1993), and as the basis of a possible experimental realization of a dynamo (Gailitis \& Freiberg 1980; Gailitis et al. 1987; Gailitis 1993). Numerical simulations of dynamo action in convective flows also show magnetic fields concentrating in swirling downdraughts (e.g. Brandenburg et al. 1990), reminiscent of Ponomarenko dynamos, although finite in the axial extent.

Flows of this family are probably the simplest kinematic dynamos known and, in the form where $\omega(r)$ and $w(r)$ are piecewise constant, were some of the earliest to be analysed mathematically (Ponomarenko 1973). Numerical studies have been conducted by Solovyev (1985a,b,c, 1987), Ruzmaikin et al. (1989), Lupian \& Shukurov 
(1992) and Léorat (1994). In the limit of high electrical conductivity the kinematic behaviour of the field can be analysed asymptotically (Ruzmaikin, Sokoloff \& Shukurov 1988; Gilbert 1988a). In fact they are basic members of a very wide class of slow dynamos (Soward 1990), including models of Braginsky (1964a,b). Of course flows such as (1.1) can also be unstable to Taylor-Couette instabilities (e.g. Stuart 1963), but in this paper we are only interested in the development of magnetic fields; so we disregard purely hydrodynamical instabilities. We remark that this could perhaps make it difficult to verify our results numerically or experimentally if our solutions only exist in regimes of parameter space for which the flow is hydrodynamically unstable. On the other hand, it is plausible that for certain parameter combinations the flow is both Taylor-Couette and magnetically stable. Further work would be required in order to determine which of these possibilities occurs in practice.

The properties of these flows as kinematic dynamos are largely understood. However little is known about how an unstable seed magnetic field eventually equilibrates through the nonlinear effects of the Lorentz force on the fluid flow: it is this we investigate, at large magnetic Reynolds number $R m$ and Reynolds number $R e$, given by

$$
R m \equiv 1 / \varepsilon=U L / \eta, \quad R e \equiv 1 / \delta=U L / v,
$$

where $U$ and $L$ are typical velocity and length scales of the flow, $\eta$ is the magnetic diffusivity, and $v$ the viscosity. It is also useful to define the magnetic Prandtl number

$$
\operatorname{Pr}_{M}=v / \eta \equiv R m / R e,
$$

as our analysis will apply for $R m$ and $R e$ with $R e \gg R m \gg 1$ (i.e. $\operatorname{Pr}_{M} \ll 1$ ), an ordering appropriate to both the solar dynamo and the geodynamo.

The axisymmetric flows (1.1) have a number of advantages for studying general questions of dynamo saturation, about which little is known. Dynamo action is obtained in flows of the form (1.1) with no need for an imposed alpha effect, or other parameterized transport effects (see e.g. Moffatt 1978). An alpha effect can be identified - the diffusion of field in curved geometry (see Soward 1990) - but this arises in the primitive equations, and is not externally imposed, there being no free parameters and no arbitrary functional forms. Because of this there are no uncertainties as to how the alpha effect is suppressed in the presence of strong fields, whereas when the alpha effect is imposed one usually has to guess how it will be suppressed. The dynamo operates by a combination of an omega effect, that is differential rotation in the flow (1.1), and this alpha effect from diffusion in curved geometry. It can thus be thought of as a dynamo of alpha-omega type.

Another advantage is that flows of the Ponomarenko family are easily driven by imposing appropriate boundary conditions, for example by placing the fluid between two infinite rotating and translating cylinders. The fluid can then adopt a spiral Couette flow, which is of the form (1.1) and is a dynamo at sufficiently large $\mathrm{Rm}$ (Solovyev 1985a,b,c, 1987). The driving through boundary conditions is attractive mathematically. Although driving of a flow by thermal convection is probably more desirable for many astrophysical applications, it leads to additional complications.

At high $R m$ and high $R e$ it is perhaps at first sight surprising that any analysis of equilibrated dynamos in this geometry is possible, and needless to say, our ambitions have to be somewhat modest. In fact for small $\operatorname{Pr}_{M}$ the problem may be reduced to a system of nonlinear ODEs depending solely on radius $r$ under certain mild assumptions discussed later; these describe steadily rotating states, which we often refer to as 'steady states' for brevity. We sketch how this is achieved. Starting with a flow and field given by (1.1) and (1.2) above, in nonlinear regimes the field will 
generally depend on three variables $(r, m \theta+k z, t)$. However we seek steadily rotating states, in which case the dependence is reduced to just two variables $(r, m \theta+k z+\varpi t)$, where $\varpi$ is the frequency and becomes an eigenvalue of the problem.

The key simplification, however, is that at large $R e$ (holding $R m$ and other parameters fixed) the dominant feedback from a magnetic mode of the form (1.2) is on the mean flow (1.1), and this is always sufficient to saturate a growing field. The field does of course also generate fluctuating flows, with modes proportional to $\exp ( \pm 2 \mathrm{i} m \theta \pm 2 \mathrm{i} k z)$, but this is a subdominant effect in a nearly inviscid rotating flow of the form (1.1). Thus at leading order no non-axisymmetric modes are excited in the flow, and so no modes other than those in (1.2) are created in the field. When seeking steadily rotating states, the problem becomes a nonlinear eigenproblem involving ODEs for the three complex fields $(a(r), b(r), c(r))$ and two real fields $(v(r), w(r))$, with $\varpi$ as an eigenvalue (\$2.2). This is valid when the limit of large $R e$ is taken with $R m$ held fixed, and we refer to this as the 'mean-flow system'. (A similar system was studied by Gilbert 1988b; however there it was not justified as a rational approximation.) Detailed justification for these assertions is presented later.

The nonlinear system involves essentially two important parameters, $m$ and $R m$ ( $R e$ may be scaled out), and we are faced with the question of how best to analyse it. The method we shall use is to start with the standard machinery of weakly nonlinear analysis to establish the amplitude and structure of solutions near a bifurcation, and then to extend the solution branch numerically and asymptotically into more strongly nonlinear regimes. Perhaps the most natural starting point would be to imagine increasing $\mathrm{Rm}$ from zero, corresponding to an experimentalist increasing the velocities of the boundaries, until the bifurcation of the first magnetic mode, with $m=O(1)$. This solution branch would then be followed for increasing $R m$ and its structure for high $R m$ elucidated. The problem with this approach, however, is that when an $m=O(1)$ mode bifurcates, its structure cannot be obtained analytically (only numerically), since $R m=O(1)$ and so large- $R m$ asymptotics do not apply. Thus we shall not take this route: we shall ultimately find the structure of the equilibrated $m=O(1)$ mode at high $R m$ by an analytically more tractable if somewhat less direct method.

To use analytical approximations for the field modes we have to take $R m \gg 1$ from the start; we could then imagine an experimentalist setting up the flow and introducing a seed magnetic field. However at large $R m$ we are faced with the problem that there is a large range of unstable modes, $1 \leqslant m \leqslant m_{\text {crit }} \gg 1$. Their growth rates are shown schematically as a function of $m$ in figure $1(a)$. The critical mode at high $R m$ has large wavenumber $m_{c r i t}=O\left(R \mathrm{~m}^{1 / 3}\right)$ and the linearly most unstable mode $m=m_{\max }$ also resides within the same scaling regime (see $\$ 4.1$ ). Mathematically we can start with the flow, introduce just one magnetic mode with a given $m$, and observe how it equilibrates at high $R \mathrm{~m}$; we can thus trace a branch of equilibrated states of varying amplitude, one for each unstable mode, parameterized by $m$ (figure $1 b$ ).

Now although the most unstable mode has $m=m_{\max }=O\left(\mathrm{Rm}^{1 / 3}\right)$ according to purely linear theory, it does not follow that the equilibrated state with this value of $m$ will be most important when nonlinearity is accounted for. We shall in fact establish in this paper that modes with smaller values of $m$ equilibrate at greater amplitudes than $m=m_{\max }$. In fact the amplitude of the equilibrated state increases as $m$ is reduced from the critical value $m_{\text {crit }}$ right down to $m=1$ - a situation summarized by figure $1(b)$. Experimentally, if we begin with a high- $R m$ flow and a general seed field, then although modes with $m=O\left(R m^{1 / 3}\right)$ will initially experience very fast growth, they will soon equilibrate, whilst longer-wavelength disturbances that initially grow 


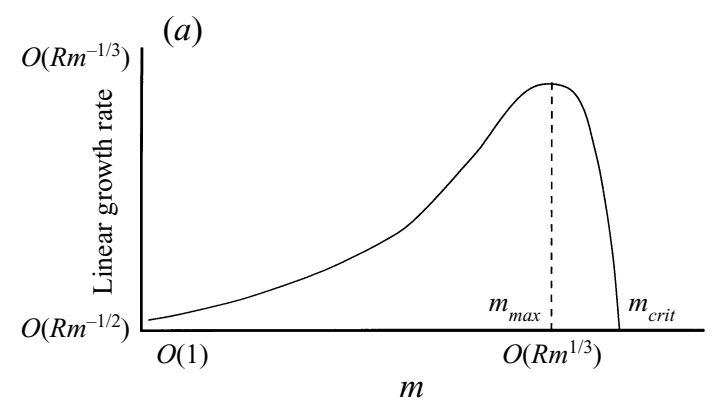

(b)

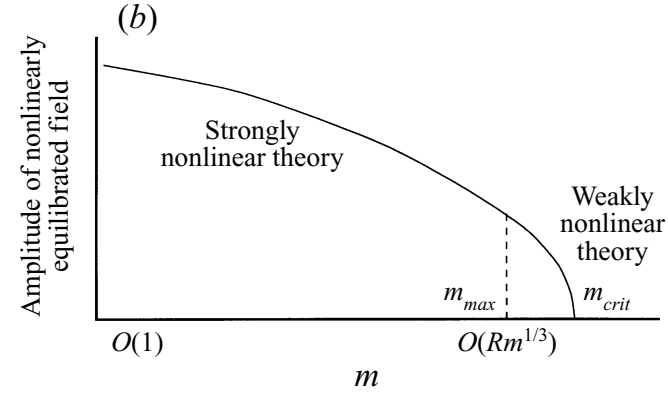

FIGURE 1. (a) Schematic picture of linear growth rates as a function of $m$ for large, fixed $R m$. (b) Schematic picture of the amplitude of a nonlinear equilibrated mode as a function of $m$ for large, fixed $\mathrm{Rm}$.

more slowly eventually settle at much larger amplitudes and so probably dominate the flow. It seems likely that dynamically the field will evolve in such an 'inverse cascade', to be dominated only by very low wavenumbers $m$. While the full dynamical evolution of the field is beyond the scope of this paper, these considerations motivate our analysis of the whole branch of equilibrated states $1 \leqslant m \leqslant m_{\text {crit }}$. To do this we fix $R m \gg 1$ and then use $m$ as a control parameter, reducing it from $m_{\text {crit }}$ down to $O(1)$ values. This procedure is mathematically convenient for us to establish the structure of this branch of equilibrated states. Of course the final results of high- $R m$ states remain valid even if other routes are taken to obtain them, for example increasing $R m$ from zero and following an $m=O(1)$ mode, as mentioned above.

We begin by formulating the governing equations for our model in $\$ \$ 2.1,2.2$, and in $\$ 2.3$ discuss the circumstances under which the flow may be described by our meanflow system; in particular we show that the dynamo cannot support so-called Taylor states. Section 3 contains the derivation of the $R m \gg 1$ nonlinear equations which are valid for wavenumbers $m=O\left(\mathrm{Rm}^{1 / 3}\right)$. Linear and weakly nonlinear analyses of these equations are possible in the vicinity of the linear critical wavenumber $m_{\text {crit }}$ and these calculations are summarized in $\S \S 4.1,4.2$. As $m$ is reduced further the amplitude of the field increases (recall figure $1 b$ ) and we follow this branch numerically in §5.1. We describe these modes as 'strongly nonlinear' and argue that this label is apt because, although at this stage the wavenumber is still $O\left(\mathrm{Rm}^{1 / 3}\right)$, the leading-order magnetic field structure is completely altered from its corresponding linear and weakly nonlinear forms.

The nature of figure $1(b)$ reminds us that as we decrease $m$ so our equilibrated amplitudes grow. It is therefore of interest to determine how far $m$ may be reduced within our assumed framework. In $\$ 5.2$ we demonstrate that it is possible to follow this strongly nonlinear mode branch into parameter regions where $m \ll O\left(R m^{1 / 3}\right)$ by 
a suitable asymptotic analysis of the nonlinear equations of $\S 3$. We prove that in this wavenumber regime the structure of the field undergoes a dramatic change: one is left with a core region in which the alpha effect operates and the motion is solid-body, bounded by thin layers in which the omega effect is dominant. We show in $\S 6$ that it is possible to obtain nonlinear equilibrated states for all values of wavenumber right down to the physically sensible limit of $m=O(1)$ so long as $R e \gg R m \gg 1$. We remark that although $\S 6$ is somewhat technical in nature, it is only at this late stage of the paper that we can pin down the exact parameter regimes for which our approach is valid. We cannot do this until the structure of the nonlinear states is known precisely and this can only be uncovered after proceeding through the stages described in $\S \S 3-5$. Furthermore, this means that a certain amount of forward referencing to $\S 6$ is inevitable within the paper, but this is a consequence of the fact that in asymptotic work one typically derives solutions under certain assumptions and then has to check a posteriori that they satisfy these constraints.

Finally, in $\$ 7$ we draw a few conclusions and finish with a short discussion of the implications of our findings. We address such topics as the level at which the magnetic field equilibrates and how the energy scales with $R m$ and $R e$. In particular, does the magnetic energy reach equipartition with the kinetic energy? Some of these issues are the subject of recent controversy (see, for example, Brandenburg et al. 1992; Vainshtein \& Cattaneo 1992; Childress \& Gilbert 1995, chapter 12 and references therein). In fact we shall see that the Ponomarenko flows are quite special as dynamos, since the streamsurfaces are cylindrical and the magnetic field saturates at levels that are viscously limited. Thus the level of the equilibrated field is probably atypically low for nonlinear dynamos. Nevertheless study of this example does give information in an area where little is known, and is a necessary first step to studying more complicated but perhaps more realistic examples.

\section{The inviscid limit and saturation through the mean flow}

\subsection{MHD equations and flow geometry}

We use the equations for incompressible MHD written in the form

$$
\begin{gathered}
\partial_{t} \boldsymbol{b}+\boldsymbol{u} \cdot \nabla \boldsymbol{b}=\boldsymbol{b} \cdot \nabla \boldsymbol{u}+\eta \nabla^{2} \boldsymbol{b}, \\
\partial_{t} \boldsymbol{u}+\boldsymbol{u} \cdot \nabla \boldsymbol{u}=\boldsymbol{b} \cdot \nabla \boldsymbol{b}-\nabla P+v \nabla^{2} \boldsymbol{u}, \\
\nabla \cdot \boldsymbol{u}=0, \quad \nabla \cdot \boldsymbol{b}=0 .
\end{gathered}
$$

In these equations units are chosen so that $\boldsymbol{b}$ has dimensions of velocity and the electric current is given by $\boldsymbol{j}=\nabla \times \boldsymbol{b}$. To obtain (2.1b) the Lorentz force $\boldsymbol{j} \times \boldsymbol{b}$ has been written as

$$
\boldsymbol{j} \times \boldsymbol{b}=\boldsymbol{b} \cdot \nabla \boldsymbol{b}-\nabla \boldsymbol{b}^{2} / 2,
$$

and the last term absorbed into the pressure $P$; the term $\boldsymbol{b} \cdot \nabla \boldsymbol{b}=\boldsymbol{F}$ is the magnetic tension force.

We prefer to work in cylindrical coordinates $(r, \theta, z)$, although the use of helical coordinates (e.g. Landman 1990; Dritschel 1991) may lead to some simplifications. We consider fluid in an infinite cylindrical shell, $r_{1}<r<r_{2}$, driven by imposing constant angular and axial velocities on the boundaries,

$$
u_{\theta}\left(r_{1}\right)=r_{1} \omega_{1}, \quad u_{\theta}\left(r_{2}\right)=r_{2} \omega_{2}, \quad u_{z}\left(r_{1}\right)=w_{1}, \quad u_{z}\left(r_{2}\right)=w_{2},
$$


and subject to no-slip boundary conditions. For the magnetic field we take perfectly conducting boundaries,

$$
b_{r}=\partial_{r} b_{\theta}+b_{\theta} / r=\partial_{r} b_{z}=0 \quad\left(r=r_{1}, r_{2}\right) .
$$

However since we will be considering only fields localized in the interior of the cylindrical shell, exponentially small at the boundaries, the details of the boundary conditions on the field will be unimportant. Overall, the choice of particular fluid and magnetic boundary conditions will have only a tiny effect on our ensuing analysis and therefore discussion of the relative merits of various boundary conditions is superfluous.

We shall have occasion to use the magnetic energy per unit length in $z$ defined by

$$
E_{M}=\frac{1}{2} \int_{r_{1}}^{r_{2}}\left\langle|\boldsymbol{b}|^{2}\right\rangle 2 \pi r \mathrm{~d} r,
$$

where $\langle\cdot\rangle$ is an average over $\theta$ and $z$. We will always be considering fields which are periodic in $z$ so that the meaning of averages over $z$ is clear. For convenience, we non-dimensionalize at the outset. Letting $U$ be a typical velocity and $L$ a typical length of the flow, which we will fix more precisely later on, we define $R m, \operatorname{Re}, \varepsilon$ and $\delta$ as in (1.3). The above equations (2.1)-(2.4) involve dimensional variables, and if we need to emphasize or distinguish these, we attach a superscript 'dim'; the quantities above could (or perhaps should) be so labelled. We then relate the dimensional quantities to dimensionless counterparts by writing

$$
\boldsymbol{r}^{d i m}=L \boldsymbol{r}, \quad \boldsymbol{u}^{d i m}=U \boldsymbol{u}, \quad t^{d i m}=(L / U) t, \quad \boldsymbol{b}^{d i m}=U \boldsymbol{b},
$$

and for geometric quantities,

$$
r_{i}^{d i m}=L r_{i}, \quad \omega_{i}^{d i m}=(U / L) \omega_{i}, \quad w_{i}^{d i m}=U w_{i} \quad(i=1,2) .
$$

Using dimensionless variables the equations for the magnetic field are now

$$
\begin{gathered}
\partial_{t} \boldsymbol{b}+\boldsymbol{u} \cdot \nabla \boldsymbol{b}=\boldsymbol{b} \cdot \nabla \boldsymbol{u}+\varepsilon \nabla^{2} \boldsymbol{b}, \\
\partial_{t} \boldsymbol{u}+\boldsymbol{u} \cdot \nabla \boldsymbol{u}=\boldsymbol{b} \cdot \nabla \boldsymbol{b}-\nabla P+\delta \nabla^{2} \boldsymbol{u},
\end{gathered}
$$

together with $(2.1 c, d),(2.3)$, which are the same in dimensionless and dimensional variables.

\subsection{Nonlinear feedback through the mean flow}

In this section we obtain equations coupling the evolution of a field mode (1.2) and the mean flow. To begin with we proceed without any approximation. First consider the flow in the absence of field, $\boldsymbol{b}=\mathbf{0}$. The unique steady solution depending only on radius $r$,

$$
\boldsymbol{u}=(0, v(r), w(r)), \quad v(r) \equiv r \omega(r),
$$

to equation $(2.8 b)$ is spiral Couette flow, which takes the form

$$
\omega=C_{1}-C_{2} / r^{2}, \quad w=C_{3}+C_{4} \log r .
$$

The constants $C_{i}$ are determined from the boundary conditions (2.3). Note that it is viscosity which controls the spiral Couette flow (2.10) and so the smaller $\delta$ is, the longer the spin-up time.

The axisymmetric flow $(2.10 a, b)$ is unstable to dynamo action provided $R m$ is large enough, and provided that the flow is sufficiently three-dimensional, in the sense that 
$\omega(r)$ and $w(r)$ are non-constant (see Solovyev 1985a,b,c, 1987; Ruzmaikin et al. 1988; Gilbert 1988a). Very weak magnetic fields $\boldsymbol{b}$ are governed solely by the linear induction equation $(2.8 a)$. We specialize to a single magnetic mode of the form (1.2), where $\varpi$ is the frequency and is real when we seek nonlinear steadily rotating states. Substituting (1.2), (2.9) into the induction equation yields

$$
\begin{aligned}
& (\mathrm{i} \varpi+\mathrm{i} m \omega+\mathrm{i} k w) a=\varepsilon\left[\nabla_{r}^{2} a-\left(\frac{m^{2}+1}{r^{2}}+k^{2}\right) a-\frac{2 \mathrm{i} m}{r^{2}} b\right], \\
& (\mathrm{i} \varpi+\mathrm{i} m \omega+\mathrm{i} k w) b=a r \partial_{r} \omega+\varepsilon\left[\nabla_{r}^{2} b-\left(\frac{m^{2}+1}{r^{2}}+k^{2}\right) b+\frac{2 \mathrm{i} m}{r^{2}} a\right], \\
& (\mathrm{i} \varpi+\mathrm{i} m \omega+\mathrm{i} k w) c=a \partial_{r} w+\varepsilon\left[\nabla_{r}^{2} c-\left(\frac{m^{2}}{r^{2}}+k^{2}\right) c\right],
\end{aligned}
$$

with $\nabla_{r}^{2} \phi \equiv r^{-1} \partial_{r}\left(r \partial_{r} \phi\right)$ as the radial part of the Laplacian operator. We may drop equation (2.11c) for $c(r)$ since for $k \neq 0$ the solenoidal condition (2.1d), which takes the form

$$
r^{-1} \partial_{r}(r a)+\mathrm{i} m r^{-1} b+\mathrm{i} k c=0,
$$

enables us to calculate $c$ from $a$ and $b$, should we require it.

The feedback on the flow from a given magnetic mode (1.2) through the tension force term $\boldsymbol{F}=\boldsymbol{b} \cdot \boldsymbol{\nabla} \boldsymbol{b}$ in $(2.8 b)$ contains a mean component, $\langle\boldsymbol{F}\rangle$, and second-harmonic flow components proportional to $\exp ( \pm 2 \mathrm{i} m \theta \pm 2 \mathrm{i} k z)$ : in $\S \S 2.3,6.2$ we shall show that when the limit $R e \rightarrow \infty$ is taken at fixed $R m$ the second harmonics may be neglected. Using integration by parts and the solenoidal condition $(2.1 d)$ the mean azimuthal and axial tension forces may be written as

$$
\left\langle F_{\theta}\right\rangle=\left\langle r^{-2} \partial_{r}\left(r^{2} b_{r} b_{\theta}\right)\right\rangle, \quad\left\langle F_{z}\right\rangle=\left\langle r^{-1} \partial_{r}\left(r b_{r} b_{z}\right)\right\rangle
$$

for general fields $\boldsymbol{b}$. The mean radial tension force can be balanced by pressure, and so need not be considered. Specializing to the one mode (1.2), we have

$$
\left\langle F_{\theta}\right\rangle=r^{-2} \partial_{r}\left[r^{2}\left(a b^{*}+a^{*} b\right)\right], \quad\left\langle F_{z}\right\rangle=r^{-1} \partial_{r}\left[r\left(a c^{*}+a^{*} c\right)\right] .
$$

Neglecting higher harmonics in the flow, the equation governing the mean flow follows from $(2.8 b)$,

$$
\begin{aligned}
-\delta r^{-2} \partial_{r}\left(r^{3} \partial_{r} \omega\right) & =r^{-2} \partial_{r}\left[r^{2}\left(a b^{*}+a^{*} b\right)\right], \\
-\delta r^{-1} \partial_{r}\left(r \partial_{r} w\right) & =r^{-1} \partial_{r}\left[r\left(a c^{*}+a^{*} c\right)\right] .
\end{aligned}
$$

Note that only viscous terms survive from $(2.8 b)$; there is no contribution from the term $\boldsymbol{u} \cdot \nabla \boldsymbol{u}$, nor from $\partial_{t} \boldsymbol{u}$ as the mean flow is constant in a steadily rotating state.

Neglect of the fluctuating component of the tension force has left us with an approximate 'mean-flow system' for the mean fluid flow (2.9) and a single magnetic field mode (1.2). Equilibrated steadily rotating states are governed by equations $(2.11 a, b)$ for the field and equations $(2.15 a, b)$ for the flow, supplemented by boundary conditions. These equations are in fact valid for any fixed $R m$, in the limit of large $R e$, as discussed in the next section. We remark that in this system the Reynolds number $R e=1 / \delta$ can be scaled away by writing equations for the scaled field $\boldsymbol{b}^{\prime}=\delta^{-1 / 2} \boldsymbol{b}$. This immediately suggests equilibration at the low level $\boldsymbol{b}=O\left(\delta^{1 / 2}\right)$ for large $R e$.

The system may be generalized to describe several magnetic modes, interacting through a single mean flow, which would be forced by a sum of terms like (2.14), one for each mode. However in this work we shall focus attention on a single magnetic mode. 


\subsection{The inviscid limit and absence of Taylor states}

We derived the mean-flow system in $\$ 2.2$ by neglecting the feedback of the field onto the second harmonic of the flow. This is a valid approximation as $R e \rightarrow \infty$ for fixed $R m$ under some mild assumptions. The justification for our approximation lies in the fact that the equations $(2.15 a, b)$ for the mean flow involve a balance between the mean tension force and only viscous terms. The net torque (and net axial force) on an infinitesimal cylinder of fluid between $r$ and $r+\mathrm{d} r$ can only be balanced by viscous stress. As $R e \rightarrow \infty$ a given mean tension force drives a large mean flow, and this is the dominant feedback mechanism. On the other hand for the second (and higher) harmonics, the tension force term can be balanced by other, non-viscous terms. Therefore for $R e \gg 1$ a magnetic field generally evokes a response from the mean flow which is nominally a factor $R e$ larger than that from the higher flow modes. (This assumes that the mean tension force and fluctuating tension force have similar magnitudes as functions of $R e$, an assumption which is valid, as discussed further below.) This indicates that the magnetic field will equilibrate at the low level $O\left(\delta^{1 / 2}\right)$, limited by viscosity.

We expand on this argument below. Similar arguments are well established within the geodynamo literature (see e.g. Roberts \& Soward 1992; Fearn 1994). To make closer contact with this work, note that we can define an Ekman number $E=v / \omega r^{2}$, using dimensional quantities, where $\omega$ is the angular velocity in the region of the flow where the magnetic field is localized. Provided $\omega \neq 0, E$ is proportional to $\delta=1 / R e$ and the limit $R e \rightarrow \infty$ then corresponds to the geophysically important limit of small Ekman number. In our problem the field then saturates at a level which is $O\left(E^{1 / 2}\right)$; the geodynamo problem in a spherical shell is complicated by Ekman layers on the boundaries, but similar arguments involve balancing mean forces against Ekman fluxes and lead to saturation at a level $O\left(E^{1 / 4}\right)$; the fluctuating forces are balanced against Coriolis terms and again do not play a role. The physical situation and scalings in our problem are similar to those used by Jones \& Roberts (1990) in a study of magnetoconvection in a rotating duct (see the discussion at the beginning of their $\S 4)$.

To see that it is a consistent approximation to neglect the fluctuating tension force, first assume that the magnetic field is $O\left(\delta^{1 / 2}\right)$, the mean and fluctuating tension forces are $O(\delta)$, the mean flow is $O(1)$, and the fluctuating flow is $O(\delta)$. If we take the Navier-Stokes equations $(2.1 b, c)$ and average them with $\langle\cdot\rangle$, we obtain the leading equations for the mean flow at order $\delta$ and these are precisely equations (2.15) above. (The contribution to the mean from products of fluctuating quantities is $O\left(\delta^{2}\right)$ and so a higher-order effect.)

Next we consider the fluctuating flow, specifically the second-harmonic component, $\boldsymbol{u}^{\prime} \exp (2 \mathrm{i} m \theta+2 \mathrm{i} k z+2 \mathrm{i} \varpi t)$, which is driven by the $O(\delta)$ tension force. With the orders of magnitude postulated in the last paragraph, taking the leading fluctuating part of the Navier-Stokes equation and neglecting terms smaller than $O(\delta)$, we obtain equations for $\boldsymbol{u}^{\prime}=\left(u^{\prime}, v^{\prime}, w^{\prime}\right)$ :

$$
\begin{aligned}
(2 \mathrm{i} \varpi+2 \mathrm{i} m \omega+2 \mathrm{i} k w) u^{\prime}-2 \omega v^{\prime} & =-\partial_{r} P^{\prime}+F_{r}^{\prime}, \\
(2 \mathrm{i} \varpi+2 \mathrm{i} m \omega+2 \mathrm{i} k w) v^{\prime}+2 \omega u^{\prime}+r u^{\prime} \partial_{r} \omega & =-2 \mathrm{i} m P^{\prime} / r+F_{\theta}^{\prime}, \\
(2 \mathrm{i} \varpi+2 \mathrm{i} m \omega+2 \mathrm{i} k w) w^{\prime} & +u^{\prime} \partial_{r} w \\
\partial_{r} u^{\prime}+u^{\prime} / r+2 \mathrm{i} m v^{\prime} / r+2 \mathrm{i} k w^{\prime} & =0 .
\end{aligned}
$$

Here $P^{\prime}$ and $\boldsymbol{F}^{\prime}=O(\delta)$ are the second-harmonic components of the pressure and tension force. This is essentially the Navier-Stokes equation linearized about the mean 
flow (2.9) with $O\left(\delta^{2}\right)$ viscous terms neglected. Provided these equations have a finite solution $\boldsymbol{u}^{\prime}=O(\delta)$ driven by the $O(\delta)$ tension force then our framework is consistent; difficulties would arise if this system had no solution for $\boldsymbol{u}^{\prime}$ and then viscosity would need to be reinstated, as happens for the mean flow.

Deciding whether this system can be solved for $\boldsymbol{u}^{\prime}$ has to be done later, when $\varpi$ and asymptotics of the equilibrated mean-field system are known, and we take up this issue in $§ 6.2$. Nevertheless we wish to make it plausible at this early stage that the system is generally soluble, and so we anticipate later analysis: it turns out that in the thin cylindrical 'core' region where the bulk of the magnetic field will be localized, the terms $\varpi+m \omega+k w, \partial_{r} \omega$ and $\partial_{r} w$ are negligible because the field is frozen in a flow that is approximately in solid-body rotation. The resulting system is

$$
\begin{aligned}
-2 \omega v^{\prime} & =-\partial_{r} P^{\prime}+F_{r}^{\prime}, \\
2 \omega u^{\prime} & =-2 \mathrm{i} m P^{\prime} / r+F_{\theta}^{\prime}, \\
0 & =-2 \mathrm{i} k P^{\prime}+F_{z}^{\prime}, \\
\partial_{r} u^{\prime}+u^{\prime} / r & +2 \mathrm{i} m v^{\prime} / r+2 \mathrm{i} k w^{\prime}=0 .
\end{aligned}
$$

Provided the field is localized at a radius where the flow has non-zero angular velocity, $\omega \neq 0$, which can always be arranged by suitable boundary conditions, this system may be solved for $\boldsymbol{u}^{\prime}$ and the approximation is consistent. Explicitly, $P^{\prime}$ is determined from $F_{z}^{\prime}$ using $(c)$, then $u^{\prime}$ and $v^{\prime}$ from $(a, b)$, and finally $w^{\prime}$ from $(d)$. The core region is bounded by thin diffusive layers; here the picture is a little more complicated and is discussed in $\S 6.2$.

The above discussion of saturation of field through the mean flow at low $O\left(\delta^{1 / 2}\right)$ levels determined by viscosity is based on the key assumption that the mean tension force is of similar size to the fluctuating tension force. As is discussed in the geodynamo literature, an alternative possibility at high $R e$ is that the field evolves into a 'Taylor state' (Taylor 1963; Malkus \& Proctor 1975; see also Moffatt 1978; Fearn 1994), in which the mean tension force is very small relative to the fluctuating tension force and the above arguments break down. The field grows to $O(1)$ values, and saturation occurs through higher harmonics. The requirement that the mean force on cylinders must vanish becomes the 'Taylor constraint' which determines partially the structure of the equilibrated field. Taylor states are found in magnetoconvection and in dynamos with prescribed alpha effects (see, for example, Jones \& Roberts 1990; Roberts \& Soward 1992; Fearn 1994).

Can the analogue of a Taylor state emerge from our mean-flow system? In the Taylor state the magnetic energy would remain $O(1)$ while $R e \rightarrow \infty$ and so the average azimuthal and axial forces $\left\langle F_{\theta}\right\rangle,\left\langle F_{z}\right\rangle$ would tend to zero. The first condition is familiar from discussion of the geodynamo problem in, say, spherical geometry: only viscosity can balance a mean azimuthal torque. The second condition, $\left\langle F_{z}\right\rangle=0$, arises because in infinite cylindrical geometry only viscous terms can balance a mean axial force; this condition does not arise in the spherical case since such a force can be absorbed by the boundary of the fluid.

However the analogue of a Taylor state cannot occur in the mean-flow system, as can be seen by examining its energy equation. In a steady state:

$$
0=\frac{\mathrm{d} E_{M}}{\mathrm{~d} t}=-\int_{r_{1}}^{r_{2}}\left(r \omega\left\langle F_{\theta}\right\rangle+w\left\langle F_{z}\right\rangle\right) 2 \pi r \mathrm{~d} r-\varepsilon \int_{r_{1}}^{r_{2}}\left\langle|\boldsymbol{j}|^{2}\right\rangle 2 \pi r \mathrm{~d} r .
$$

The last term represents the Ohmic dissipation, which can be bounded below by a constant times $\varepsilon E_{M}$ in the present geometry. The dissipation is balanced by the 
working of the fluid against the mean tension forces $\left\langle F_{\theta}\right\rangle$ and $\left\langle F_{z}\right\rangle$. Thus these forces cannot tend to zero as $R e \rightarrow \infty$ (with $\varepsilon$ fixed) in a steady state with $O(1)$ magnetic energy, as there would be nothing to balance Ohmic dissipation. In fact neither of these mean forces can vanish individually, as can be established by looking at the energy budget for $\left(b_{r}, b_{\theta}\right)$ components and the $b_{z}$ component separately.

We conclude that no Taylor states can emerge from the steady mean-flow/magnetic field system described above. The absence of Taylor states here is a consequence of the fact that in the mean-flow system the energy source for the field is precisely through the forces that would vanish in a Taylor state. This is property of the basic spiral Couette flow $\boldsymbol{u}(r)$ chosen, and the result that the feedback from the field preserves this simple dependence of $\boldsymbol{u}$ on $r$. This is not to say that there is no possibility of Taylor states in the full system, that is a fluid and field obeying the MHD equations and the boundary conditions $(2.3)$ - it would be unwise to speculate here given the complexity of flow that may be supported in Couette geometry with the given boundary conditions - but just that Taylor states cannot be connected to a linear regime of weak magnetic field through steady states of the mean-flow system. Put differently, at large $R e$ the mean-flow system considered here is certainly sufficient to saturate growing magnetic modes at a low level.

However, alternative routes to saturation are also plausible (and could coexist in a complicated bifurcation diagram for a highly supercritical fluid system such as this), in which non-mean modes would be excited in the flow field. In this case the fluid could adopt a flow $\boldsymbol{u}(r, \theta, z, t)$, not dependent solely on $r$ but still consistent with the boundary conditions (2.3), for example Taylor rolls. Growing magnetic fields could then perhaps adopt a Taylor state, with vanishing mean tension forces, by being supplied with energy through interaction between field modes and non-mean flow modes. In terms of the energy equation (2.18), an energy input term such as $r \omega\left\langle F_{\theta}\right\rangle$ would be replaced by $\left\langle r \omega F_{\theta}\right\rangle$ and there would be no difficulty in maintaining a Taylor state with $\left\langle F_{\theta}\right\rangle \simeq 0$. This however cannot be captured in the mean-flow system we consider.

\section{The mean-flow system at large $R m$}

\subsection{Choice of scalings}

The system (2.3), (2.11a,b), (2.12), (2.15) for mean flow and field is valid for large Re with other quantities held constant. However it is analytically intractable even for linear dynamo behaviour unless we also consider the limit of large $\mathrm{Rm}$. With two large parameters the question of their relative size, in other words the magnitude of the magnetic Prandtl number (1.4), arises. We defer addressing this point until §6.2, since the restriction, which turns out to be simply $R e \gg R m$, depends on the structure that the field adopts, an unknown at this stage. For the time being we fix $R m$ large, and then choose $R e$ sufficiently large so that the mean-flow system may be used.

In this section we derive nonlinear equations for the field taking into account feedback from the mean flow. These equations include the linear theory of Ruzmaikin et al. (1988), Gilbert (1988a) and, as motivation, we recall scalings used in Gilbert $(1988 a)$. In linear theory at high $R m=1 / \varepsilon$ the most unstable and the marginal modes have $m, k=O\left(\varepsilon^{-1 / 3}\right)$. The field for each $(m, k)$ mode is localized a distance of order $\varepsilon^{1 / 3}$ about a radius $r_{0}$ determined by a resonance condition given below in (3.6). We set

$$
r=r_{0}+\varepsilon^{1 / 3} \zeta, \quad m=\varepsilon^{-1 / 3} m_{0}, \quad k=\varepsilon^{-1 / 3} k_{0},
$$


with $\zeta, m_{0}, k_{0}$ of order one. In the linear problem the field is expanded in powers of $\varepsilon^{1 / 3}$,

$$
a(r)=\lambda\left(\varepsilon^{1 / 3} a_{0}+\varepsilon^{2 / 3} a_{1}+\cdots\right), \quad b(r)=\lambda\left(b_{0}+\varepsilon^{1 / 3} b_{1}+\cdots\right)
$$

(we do not need to consider $c(r)$, which scales and behaves similarly to $b(r)$ ). Here $\lambda$ is an amplitude which plays no role in the linear problem, but which we shall need to fix to bring in nonlinear terms at the correct level. We expand the frequency as

$$
\varpi=\varepsilon^{-1 / 3} \varpi_{0}+\varepsilon^{1 / 3} \varpi_{2}+\cdots .
$$

The first term we need to deal with is the advective term on the left-hand side of $(2.11 a, b)$. This is expanded close to $r_{0}$ in a series

$$
\begin{aligned}
\mathrm{i} \varpi+\mathrm{i} m \omega+\mathrm{i} k w= & \varepsilon^{-1 / 3}\left(\mathrm{i} \varpi_{0}+\mathrm{i} m_{0} \omega\left(r_{0}\right)+\mathrm{i} k_{0} w\left(r_{0}\right)\right)+\zeta\left(\mathrm{i} m_{0} \omega^{\prime}\left(r_{0}\right)+\mathrm{i} k_{0} w^{\prime}\left(r_{0}\right)\right) \\
& +\varepsilon^{1 / 3}\left[\mathrm{i} \varpi_{2}+\frac{1}{2} \zeta^{2}\left(i m_{0} \omega^{\prime \prime}\left(r_{0}\right)+\mathrm{i} k_{0} w^{\prime \prime}\left(r_{0}\right)\right)\right]+\cdots,
\end{aligned}
$$

with primes denoting radial derivatives. The leading constant and linear parts are eliminated by setting

$$
\mathrm{i} \varpi_{0}+\mathrm{i} m_{0} \omega\left(r_{0}\right)+\mathrm{i} k_{0} w\left(r_{0}\right)=0,
$$

which determines the leading frequency $\varpi_{0}$ as that given by advection at the radius $r_{0}$, and

$$
\mathrm{i} m_{0} \omega^{\prime}\left(r_{0}\right)+\mathrm{i} k_{0} w^{\prime}\left(r_{0}\right)=0 .
$$

This resonance condition fixes the critical radius $r_{0}$ at which the $(m, k)$ mode is localized. At this radius the shearing action of the flow is along lines of constant field, and so here the field is least vulnerable to the creation of small scales by advection and their destruction by diffusion. We assume that $r_{1}<r_{0}<r_{2}$ so that our mode is confined away from either boundary; our subsequent analysis is then largely independent of the boundary conditions imposed on the field and flow.

The next term in the expansion (3.4) of the advection operator is

$$
\varepsilon^{1 / 3}\left[\mathrm{i} \varpi_{2}+\frac{1}{2} \zeta^{2}\left(\mathrm{i} m_{0} \omega^{\prime \prime}\left(r_{0}\right)+\mathrm{i} k_{0} w^{\prime \prime}\left(r_{0}\right)\right)\right]
$$

and this gives the leading effect of the flow on the field near to $r_{0}$. This important term enters into the linear problem and the $\zeta^{2}$ dependence, which arises from differential rotation, localizes the magnetic modes in the form of parabolic cylinder functions. The real part of the frequency $\varpi_{2}$ gives the motion of field relative to the fluid flow. We will fix the amplitude $\lambda$ of the field so as to bring in nonlinear effects at the level $O\left(\varepsilon^{1 / 3}\right)$ of this term.

We now look at the nonlinear feedback on the flow from the field. Equations $(2.15 a, b)$ may be formally integrated to give

$$
\begin{gathered}
\omega=-\delta^{-1} \int_{r_{0}}^{r} r^{-1}\left(a b^{*}+a^{*} b\right) \mathrm{d} r+\tilde{\omega}(r), \\
w=-\delta^{-1} \int_{r_{0}}^{r}\left(a c^{*}+a^{*} c\right) \mathrm{d} r+\tilde{w}(r) .
\end{gathered}
$$

The integrals are particular integrals, giving localized feedback on the flow from the field, while the terms $\tilde{\omega}$ and $\tilde{w}$ are complementary functions, solutions of the homogeneous equations of the form

$$
\tilde{\omega}=C_{1}-C_{2} / r^{2}, \quad \tilde{w}=C_{3}+C_{4} \log r .
$$


The four constants $C_{1}-C_{4}$ are determined by the boundary conditions (2.3); for example, $C_{1}$ and $C_{2}$ are given by the simultaneous equations

$$
\begin{aligned}
& \delta^{-1} \int_{r_{1}}^{r_{0}} r^{-1}\left(a b^{*}+a^{*} b\right) \mathrm{d} r+C_{1}-C_{2} / r_{1}^{2}=\omega_{1}, \\
& -\delta^{-1} \int_{r_{0}}^{r_{2}} r^{-1}\left(a b^{*}+a^{*} b\right) \mathrm{d} r+C_{1}-C_{2} / r_{2}^{2}=\omega_{2},
\end{aligned}
$$

and similar equations hold for $C_{3}$ and $C_{4}$. For zero magnetic field these, with (3.8), (3.9), give the original spiral Couette flow but the presence of magnetic field leads to a modification of the constants and hence an adjustment of the whole flow field. Fortunately we shall see that this 'global adjustment', which is sensitive to the detailed boundary conditions imposed, is small and may be neglected.

The flow affects the field in equations $(2.11 a, b)$ through just two terms. The first of these is the azimuthal stretching term,

$$
r \partial_{r} \omega=-\delta^{-1}\left(a b^{*}+a^{*} b\right)+r \partial_{r} \tilde{\omega},
$$

from $(3.8 a)$. The second is the advection term,

$$
\mathrm{i} m \omega+\mathrm{i} k w=-\delta^{-1} \int_{r_{0}}^{r}\left(a \partial_{r} a^{*}-a^{*} \partial_{r} a\right) \mathrm{d} r+\mathrm{i} m \tilde{\omega}+\mathrm{i} k \tilde{w} ;
$$

this has been rewritten in terms of $a$ using $(3.8 a, b)$ and the condition $\nabla \cdot \boldsymbol{b}=0,(2.12)$ and is exact. With the scalings (3.2) the integral in (3.12) is of order $\lambda^{2} \varepsilon^{2 / 3} / \delta$ and varies on the short $\zeta$-scale. This term becomes important in nonlinear saturation when it is of the same size as the key advection term (3.7), which is of size $\varepsilon^{1 / 3}$. To bring in both simultaneously we should set $\lambda^{2} \varepsilon^{2 / 3} / \delta=\varepsilon^{1 / 3}$, that is

$$
\lambda^{2}=\delta \varepsilon^{-1 / 3} .
$$

What are the consequences of fixing the field amplitude at this level? The first is that the magnetic field term in (3.11) is of order one, and so the shear (3.11) in the layer is modified at the same time as the advection term (3.12). These two nonlinear effects, modification of advection and shear, both play a role in the nonlinear equilibration.

A second effect is that the global adjustment to the flow field is small and ultimately unimportant; the integral terms in (3.10) are a factor $\varepsilon^{1 / 3}$ smaller than the other terms and so lead to $O\left(\varepsilon^{1 / 3}\right)$ adjustments to the $C_{i}$. The result is to detune slightly the condition (3.6) which fixes the location of the layer and so, as the field grows, the centre of the layer moves a distance $O\left(\varepsilon^{1 / 3}\right)$ in terms of $r$ or $O(1)$ in terms of $\zeta$. Since it is convenient to have the layer centred at $\zeta=0$, one option is to translate the origin of $\zeta$ to follow the location of the layer as the amplitude of the field is increased.

However, a rather easier alternative is to adjust $m$ and $k$, which define the layer location, so as to keep the layer centred at our original choice $\zeta=0$. To do this formally in the expansion would involve expanding each constant $C_{i}$ in (3.9) as a power series in $\varepsilon^{1 / 3}$. This allows us to keep track of flow adjustment and if $m, k$ and $\varpi$ are also expanded in this way then the constant terms in $i \varpi+i m \tilde{\omega}+\mathrm{i} k \tilde{w}$ at orders $\varepsilon^{-1 / 3}$ and 1 , together with the linear terms at orders 1 and $\varepsilon^{1 / 3}$, can be eliminated. When this procedure is implemented the results are equivalent to ignoring completely the global adjustment to the flow and, for simplicity, we shall do this. In other words, $\tilde{\omega}$ and $\tilde{w}$ are now taken to be given by (3.9) with

$$
C_{1}-C_{2} / r_{i}^{2}=\omega_{i}, \quad C_{3}+C_{4} \log r_{i}=w_{i} \quad(i=1,2)
$$


fixed independent of field magnitude. Note that the small adjustment to the flow and the handling of this are sensitive to the type of boundary conditions employed. On the other hand, once the layer is recentred at $\zeta=0$, the equations obtained are independent of the details of boundary conditions.

\subsection{Rescaling and non-dimensionalization}

Following these preparatory remarks we can now expand and obtain the nonlinear equations that are the subject of this paper. We scale $r, m$ and $k$ as in (3.1) and $\varpi$ as in (3.3), and from (3.2), (3.13) scale the field as

$$
\begin{aligned}
& a=\delta^{1 / 2} \varepsilon^{1 / 6}\left(a_{0}+\varepsilon^{-1 / 3} a_{1}+\cdots\right), \\
& b=\delta^{1 / 2} \varepsilon^{-1 / 6}\left(b_{0}+\varepsilon^{-1 / 3} b_{1}+\cdots\right) .
\end{aligned}
$$

Substituting into $(2.11 a, b),(3.11),(3.12)$, at $O\left(\varepsilon^{-1 / 3}\right)$ and $O(1)$ we obtain again

$$
\mathrm{i} \varpi_{0}+\mathrm{i} m_{0} \tilde{\omega}\left(r_{0}\right)+\mathrm{i} k_{0} \tilde{w}\left(r_{0}\right)=0, \quad \mathrm{i} m_{0} \tilde{\omega}^{\prime}\left(r_{0}\right)+\mathrm{i} k_{0} \tilde{w}^{\prime}\left(r_{0}\right)=0 .
$$

At $O\left(\varepsilon^{1 / 3}\right)$ we obtain the equations for the magnetic field which can be cast in the form

$$
\hat{\mathscr{L}} a_{0}=2 \mathrm{i} m_{0} b_{0} / r_{0}^{2}, \quad \hat{\mathscr{L}} b_{0}=\left(a_{0} b_{0}^{*}+a_{0}^{*} b_{0}-\rho\right) a_{0},
$$

with a scalar advection-diffusion operator defined as

$$
\hat{\mathscr{L}}=\partial_{\zeta}^{2}-\frac{m_{0}^{2}\left(1+\sigma^{2}\right)}{r_{0}^{2}}-\mathrm{i}\left(\varpi_{2}+\frac{m_{0} \zeta^{2} \rho \chi}{2 r_{0}^{2}}+\mathrm{i} \int_{0}^{\zeta}\left(a_{0} \partial_{\zeta} a_{0}^{*}-a_{0}^{*} \partial_{\zeta} a_{0}\right) \mathrm{d} \zeta\right) .
$$

Here the real part of the operator $\hat{\mathscr{L}}$ contains leading-order diffusion effects from (2.11), with $k_{0}$ eliminated in favour of $m_{0}$ using $(3.16 b)$. The imaginary part of $\hat{\mathscr{L}}$ gives the advection of the field in the flow local to $r_{0}$. New constant quantities describing this local flow structure have been introduced:

$$
\rho=r_{0} \tilde{\omega}^{\prime}\left(r_{0}\right), \quad \sigma=\frac{r_{0} \tilde{\omega}^{\prime}\left(r_{0}\right)}{\tilde{w}^{\prime}\left(r_{0}\right)}, \quad \chi=\left.r_{0}\left(\frac{\tilde{\omega}^{\prime \prime}}{\tilde{\omega}^{\prime}}-\frac{\tilde{w}^{\prime \prime}}{\tilde{w}^{\prime}}\right)\right|_{r=r_{0}} .
$$

The number of parameters can be reduced by pinning down our original nondimensionalization more precisely. If we define $L$ and $U$ in terms of the dimensional values of the radius at which the mode is located and the azimuthal shear at this radius then

$$
L=r_{0}^{d i m}, \quad U=\left.\left(r^{d i m}\right)^{2}\left(\tilde{\omega}^{d i m}\right)^{\prime}\right|_{r^{\text {dim }}=r_{0}^{d i m},}
$$

and, in terms of dimensionless variables, we have $r_{0}=1$ and $\rho=1$. Furthermore for spiral Couette flow $\chi \equiv-2$.

With these results, and on writing $A=a_{0}, B=b_{0}, M=m_{0}, \Omega=\varpi_{2}$, the system governing the magnetic field may be written

$$
\mathscr{L} A=2 \mathrm{i} M B, \quad \mathscr{L} B=(\beta-1) A .
$$

The operator $\mathscr{L}$ is given by

$$
\mathscr{L}=\partial_{\zeta}^{2}-M^{2}\left(1+\sigma^{2}\right)+\mathrm{i}\left(M \zeta^{2}-\alpha-\Omega\right)
$$

and the nonlinear feedback is through the real quantities

$$
\alpha(\zeta)=\mathrm{i} \int_{0}^{\zeta}\left(A \partial_{\zeta} A^{*}-A^{*} \partial_{\zeta} A\right) \mathrm{d} \zeta, \quad \beta(\zeta)=A B^{*}+A^{*} B .
$$


This is the nonlinear system appropriate to saturation of dynamo instabilities, which we will explore. On the left-hand sides of $(3.21 a, b)$ are scalar advection-diffusion operators $\mathscr{L}$, which in isolation would give decay of field. The terms on the right of these equations, however, arise from the vector nature of the field. The term $(\beta-1) A$ on the right of $(3.21 b)$ represents the omega effect: the stretching of radial $A$-field to give $B$-field by differential rotation. This is the only energy source in the problem.

The term on the right of $(3.21 a)$ is the alpha effect mentioned in the introduction (note this has no relation with the quantity called ' $\alpha$ '). It arises because in cylindrical geometry diffusion of a vector quantity such as magnetic field couples $r$ - and $\theta$-components. This term then regenerates $A$-field from $B$-field and, although it does not provide energy, is an essential ingredient in the dynamo instability. Being purely geometrical, it is not nonlinearly suppressed (unlike the omega effect). The combination of alpha effect and omega effect allows exponential growth of weak fields, as discussed in $\$ 4.1$ below.

The system can be thought of as a thin-layer approximation in cylindrical geometry yielding a planar system; the only effect arising from curvature in the original system is the alpha-effect term on the right-hand side of (3.21a). The parameter $\sigma$ is the only remnant of the flow geometry and records the local helical pitch of streamlines. In what follows the scaled wavenumber $M$ will be treated as a control parameter (for reasons discussed in the introduction) and we remark that $\Omega$ is the scaled frequency of rotation of the magnetic mode relative to the flow field at $r=r_{0}$.

\section{Linear and weakly nonlinear theory}

\subsection{Linear theory}

Linear theory at small field amplitudes is recovered by setting the nonlinear terms $\alpha$ and $\beta$ in (3.21) to zero, to give

$$
\mathscr{L}_{\text {lin }} A=2 \mathrm{i} M B, \quad \mathscr{L}_{\text {lin }} B=-A,
$$

with

$$
\mathscr{L}_{\text {lin }} \equiv \partial_{\zeta}^{2}+\mathrm{i} M \zeta^{2}-\mathrm{i} \hat{\Omega}, \quad \mathrm{i} \hat{\Omega} \equiv \mathrm{i} \Omega_{\text {lin }}+M^{2}\left(1+\sigma^{2}\right) .
$$

The frequency, $\Omega \equiv \Omega_{\operatorname{lin}}(M)$ is complex for a growing mode. The linear theory for this system is given in Ruzmaikin et al. (1988) and Gilbert (1988a); we summarize it here briefly. If $B$ is eliminated from $(4.1 a, b)$, we are left with $\mathscr{L}_{\operatorname{lin}}^{2} A=-2 \mathrm{i} M A$, which can be factorized to give

$$
\mathscr{L}_{\text {lin }}^{+} \mathscr{L}_{\text {lin }}^{-} A=0, \quad \mathscr{L}_{\text {lin }}^{ \pm} \equiv \mathscr{L}_{\text {lin }} \pm(-2 \mathrm{i} M)^{1 / 2} .
$$

The two operators $\mathscr{L}_{\text {lin }}^{ \pm}$differ by a constant and so commute; therefore the general solution is $A=\phi^{+}+\phi^{-}$, where $\phi^{ \pm}$are arbitrary solutions of $\mathscr{L}_{\operatorname{lin}}^{ \pm} \phi^{ \pm}=0$. The operators can be written in the canonical form by setting $s=\zeta(-4 \mathrm{i} M)^{1 / 4}$ :

$$
\mathscr{L}_{\text {lin }}^{ \pm}=(-4 \mathrm{i} M)^{1 / 2}\left[\partial_{s}^{2}-s^{2} / 4-\left(\mathrm{i} \hat{\Omega} \mp(-2 \mathrm{i} M)^{1 / 2}\right) /(-4 \mathrm{i} M)^{1 / 2}\right] .
$$

Solutions of $\mathscr{L}_{\text {lin }}^{ \pm} \phi^{ \pm}=0$ can be expressed in terms of parabolic cylinder functions and, in order that the mode is effectively confined to the thin layer surrounding $r=r_{0}$, we need solutions which decay as $s \rightarrow \pm \infty$. Such solutions exist provided 
$\mathrm{i} \hat{\Omega} \mp(-2 \mathrm{i} M)^{1 / 2}=-(n+1 / 2)(-4 \mathrm{i} M)^{1 / 2}$ for $n=0,1, \ldots$ and the corresponding linear growth rates are then given by

$$
\mathrm{i} \Omega_{\text {lin }}(M)=(-2 \mathrm{i} M)^{1 / 2}\left[ \pm 1-\sqrt{2}\left(n+\frac{1}{2}\right)\right]-M^{2}\left(1+\sigma^{2}\right)
$$

(from $4.1 d$ ). The real and imaginary parts of the growth rate are, respectively,

$$
\begin{aligned}
& \operatorname{Re} \mathrm{i} \Omega_{\text {lin }}(M)=M^{1 / 2}\left[ \pm 1-\sqrt{2}\left(n+\frac{1}{2}\right)\right]-M^{2}\left(1+\sigma^{2}\right), \\
& \operatorname{Im} \mathrm{i} \Omega_{\text {lin }}(M)=M^{1 / 2}\left[\mp 1+\sqrt{2}\left(n+\frac{1}{2}\right)\right] .
\end{aligned}
$$

If we translate into dimensional units we find that, to this order, equations (3.3), (3.20) give a real growth rate of

$$
\operatorname{Re} \mathrm{i} \varpi \simeq\left(\eta m \omega^{\prime}\left(r_{0}\right) / r_{0}\right)^{1 / 2}\left[ \pm 1-\sqrt{2}\left(n+\frac{1}{2}\right)\right]-\eta\left(m^{2} / r_{0}^{2}+k^{2}\right),
$$

for $n=0,1, \ldots$. These agree with growth rates obtained for more general axisymmetric flows by Ruzmaikin et al. (1988) and Gilbert (1988a).

Of these two infinite families of solutions, only the upper sign in (4.5) and the choice $n=0$ imply a positive growth rate for spiral Couette flow. Therefore we need only work with this case, for which the corresponding eigenfunctions are

$$
A_{\text {lin }}=\exp \left[-\zeta^{2}(1-\mathrm{i})(M / 8)^{1 / 2}\right], \quad B_{\text {lin }}=\frac{1}{2} M^{-1 / 2}(1+\mathrm{i}) A_{\text {lin }} .
$$

Our scaling of the original wavenumber $m=\varepsilon^{-1 / 3} M$ for small $\varepsilon$ captures both the most unstable mode and the critical mode $M=M_{\mathrm{c}}$ with

$$
M_{c}^{3 / 2} \equiv(1-1 / \sqrt{2})\left(1+\sigma^{2}\right)^{-1} .
$$

Modes with $1 \leqslant m=o\left(\varepsilon^{-1 / 3}\right)$ are also unstable, and have growth rates given by (4.6), but now the last term may be neglected. The growth rate is shown schematically as a function of $m$ in figure $1(a)$.

\subsection{Weakly nonlinear theory}

The evolution of a mode close to the bifurcation at $M=M_{c}$ can be determined by weakly nonlinear theory, which we now sketch (see also Gilbert 1988b). We write $A$ and $B$ at leading order as the linear eigenfunctions (4.7) at the bifurcation, multiplied by a small real amplitude $\Delta$. We use a subscript ' $c$ ' to denote linear eigenfunctions and other quantities at the critical point. Because the feedback from the tension force is quadratic in the field, we expand

$$
\begin{gathered}
(A, B)=\Delta\left(A_{c}, B_{c}\right)+\Delta^{3}\left(A^{\prime}, B^{\prime}\right)+O\left(\Delta^{5}\right), \\
M=M_{c}+\Delta^{2} M^{\prime}+O\left(\Delta^{4}\right), \quad(\alpha, \beta)=\Delta^{2}\left(\alpha^{\prime}, \beta^{\prime}\right)+O\left(\Delta^{4}\right) .
\end{gathered}
$$

We take the frequency $\Omega$ in the governing system (3.21) to be purely real and seek steadily rotating states with

$$
\Omega=\Omega_{c}+\Delta^{2} \Omega^{\prime}+O\left(\Delta^{4}\right) .
$$

We substitute these expansions in the nonlinear equations (3.21). At leading order in $\Delta$ we obtain exactly the linear equations (4.1) at $M_{c}$, which are automatically satisfied because of the choice of leading terms in the expansions (4.9), (4.10). The correction terms $\left(A^{\prime}, B^{\prime}\right)$ to the magnetic field are determined by the system

$$
\mathscr{L}_{c} A^{\prime}+\mathscr{L}^{\prime} A_{c}=2 \mathrm{i} M_{c} B^{\prime}+2 \mathrm{i} M^{\prime} B_{c}, \quad \mathscr{L}_{c} B^{\prime}+\mathscr{L}^{\prime} B_{c}=\beta^{\prime} A_{c}-A^{\prime},
$$




$$
\mathscr{L}^{\prime} \equiv-2 M_{c} M^{\prime}\left(1+\sigma^{2}\right)+\mathrm{i}\left(M^{\prime} \zeta^{2}-\alpha^{\prime}-\Omega^{\prime}\right) .
$$

Here $\mathscr{L}_{c}$ is the linear operator (4.1c) at the critical point; $\alpha^{\prime}$ and $\beta^{\prime}$ are obtained by substituting $A_{c}$ and $B_{c}$ in equations $(3.21 d, e)$.

We define an inner product by $\langle(A, B),(C, D)\rangle=\int\left(A^{*} C+B^{*} D\right) \mathrm{d} \zeta$ and let $\left(A^{\dagger}, B^{\dagger}\right)$ be the eigenfunction of the adjoint system to (4.1) at the critical point, given by

$$
\mathscr{L}_{c}^{\dagger} A^{\dagger}+B^{\dagger}=0, \quad \mathscr{L}_{c}^{\dagger} B^{\dagger}+2 i M_{c} A^{\dagger}=0, \quad \mathscr{L}_{c}^{\dagger} \equiv \partial_{\zeta}^{2}-\mathrm{i} M_{c} \zeta^{2}+\mathrm{i} \hat{\Omega}_{c}^{*} .
$$

It may be easily checked that the adjoint eigenfunction is given in terms of the direct eigenfunction (4.7) by $\left(A^{\dagger}, B^{\dagger}\right)=\left(B_{c}^{*}, A_{c}^{*}\right)$. Following standard methods, we take the inner product of the system $(4.11 a, b)$ with the adjoint eigenfunction to give a solvability condition which determines $M^{\prime}$ and $\Omega^{\prime}$ as

$$
M^{\prime} \simeq-0.45846 M_{c}^{1 / 2}, \quad \Omega^{\prime} \simeq 0.30834
$$

where $M_{c}$ is as given by (4.8).

\section{Strongly nonlinear states}

Having dealt with equilibration near the bifurcation $M=M_{c}$ using weakly nonlinear theory, we pursue this branch into more strongly nonlinear regimes with $M<M_{c}$ first by means of numerical computations, then by asymptotic analysis.

\subsection{Numerical results}

We work with the system (3.21). It was solved numerically using a standard nonlinear eigenvalue solver from the NAG suite of routines. It was found to be best to rewrite the system as eleven real first-order equations with two of the variables taken to be the scaled wavenumber $M$ and the frequency $\Omega$. Based on the linear results described in $\$ 4.1$ it is apparent that the most dangerous mode is an even function about $\zeta=0$ and hence computations were restricted to the semi-infinite domain $\zeta \geqslant 0$ with appropriate symmetry constraints imposed on $\zeta=0$.

Rather than solve (3.21) for prescribed $M$, it was found more convenient to specify the value of the $A$-component of field on the centreline $\zeta=0$ by setting $A(0)=\Delta$, with $\Delta$ taken to be real since the phase of the field can be fixed arbitrarily. In view of the symmetry properties we solved (3.21) subject to

$$
A^{\prime}(0)=B^{\prime}(0)=0, \quad A(0)=\Delta, \quad A(\zeta), B(\zeta) \rightarrow 0 \text { as } \zeta \rightarrow \infty .
$$

The numerical code then found $M$ and $\Omega$ as functions of amplitude $\Delta$, together with the structure of the nonlinear eigenfunctions. As a starting point we used small values of $\Delta$ and checked our code by comparing its results to the weakly nonlinear predictions of $\S 4.2$ : excellent agreement with the results (4.9), (4.14) was achieved for sufficiently small $\Delta$.

Results for steady states of the strongly nonlinear system are listed in table 1 for various values of $\Delta$ up to 5 and the helical pitch parameter $\sigma$ set at unity. From table 1 we conclude that as $\Delta$ rises so $M$ falls, as is suggested in figure $1(b)$. The corresponding eigenfunctions are shown in figure 2 for the representative choices $\Delta=0.5,1,2$ and 4 . In figure $2(a)$, with $\Delta=0.5$, the field structure is not dissimilar to the linear eigenfunctions given by (4.7). As $\Delta$ grows so the field approaches a form which is characterized by a well-defined 'core' region in which the field strength is appreciable, supplemented by some type of thin 'diffusive layer' which reduces the disturbance to practically zero in an outer region. It is evident from figure $2(c, d)$ that 


\begin{tabular}{ccccccc}
\hline$\Delta$ & $M$ & $\Omega$ & $\operatorname{Re} B(0)$ & $\operatorname{Im} B(0)$ & $\alpha(\infty)$ & $\zeta_{0}$ \\
0.5 & 0.21721 & -0.0858 & 0.41631 & 0.43974 & 0.24426 & 3.25 \\
1.0 & 0.08292 & -0.0034 & 0.47752 & 0.73015 & 0.83114 & 5.00 \\
1.5 & 0.03346 & $1.3 \times 10^{-5}$ & 0.33296 & 0.87687 & 1.35984 & 8.10 \\
2.0 & 0.01832 & $7.9 \times 10^{-7}$ & 0.24996 & 0.95680 & 1.81531 & 11.65 \\
2.5 & 0.01161 & $8.1 \times 10^{-8}$ & 0.19999 & 1.01259 & 2.23541 & 15.55 \\
3.0 & 0.00803 & $1.3 \times 10^{-8}$ & 0.16666 & 1.05647 & 2.63289 & 19.80 \\
3.5 & 0.00589 & $2.7 \times 10^{-9}$ & 0.14286 & 1.09319 & 3.01384 & 24.35 \\
4.0 & 0.00451 & $7.2 \times 10^{-10}$ & 0.12500 & 1.12507 & 3.38182 & 29.14 \\
4.5 & 0.00356 & $2.2 \times 10^{-10}$ & 0.11111 & 1.15341 & 3.73918 & 34.18 \\
5.0 & 0.00288 & $8.0 \times 10^{-11}$ & 0.10000 & 1.17904 & 4.08758 & 39.45
\end{tabular}

TABLE 1. Results of simulations of (3.21), (5.1); $\zeta_{0}$ is the leading zero of $\operatorname{Re} A(\zeta)$.
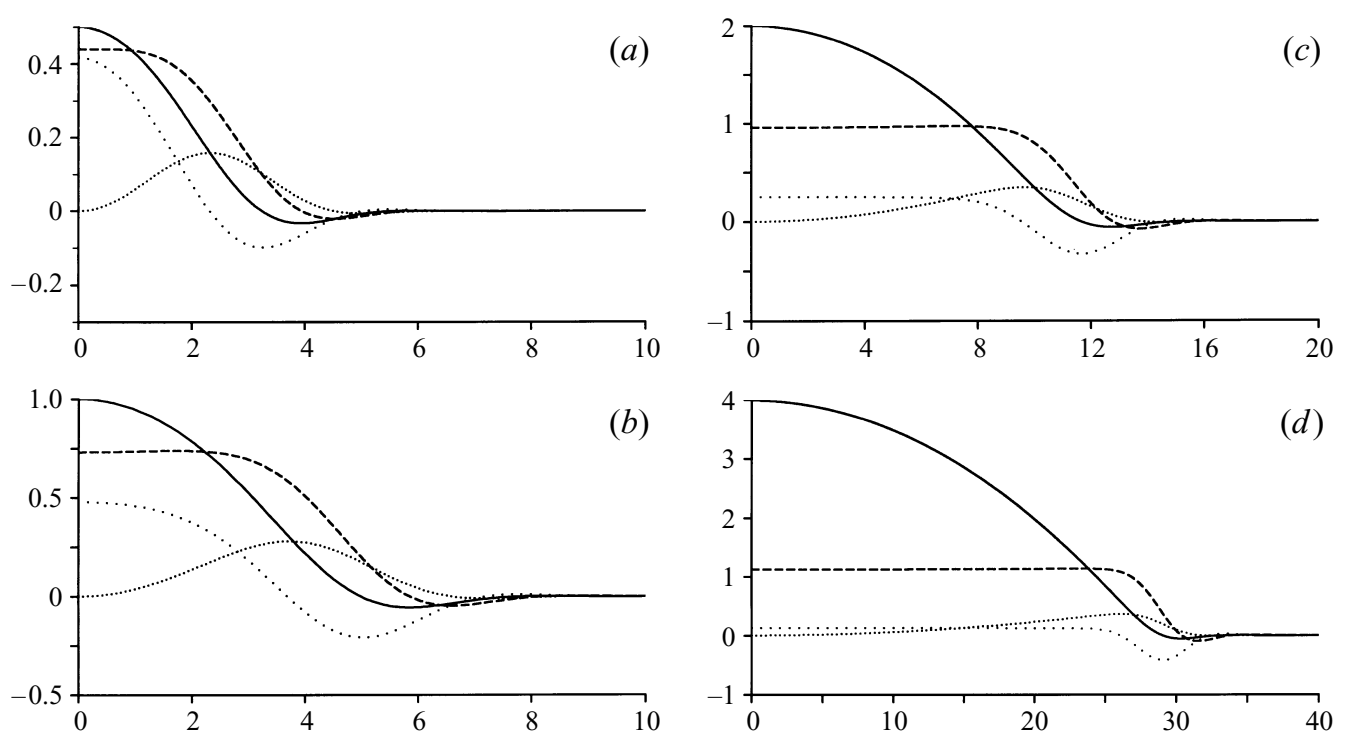

FiguRE 2. Fields in equilibrated nonlinear states with $\sigma=1$. Shown are the fields $\operatorname{Re} A$ (solid), $\operatorname{Im} A$ (dotted), $\operatorname{Re} B$ (wide dotted) and $\operatorname{Im} B$ (dashed), solutions of (3.21), plotted as functions of $\zeta$. In $(a-d) \Delta=0.5,1,2$ and 4 respectively.

in the core $\operatorname{Re} A$ takes a parabolic shape, $\operatorname{Im} B$ is virtually constant and $\operatorname{Im} A$ and $\operatorname{Re} B$ are quite small. One distinctive feature is that for some reason $|\operatorname{Re} B|$ actually appears to be larger within the diffusive layer than outside and it decays both into the core and the outer zones.

The behaviour of the nonlinear terms $\alpha$ and $\beta$ (see $(3.21 d, e)$ ) is plotted in figure 3 . This shows $\alpha(\zeta) / \Delta$ (solid) and $1-\beta(\zeta)$ (dotted) for the parameter values corresponding to those in figure 2 . We see that, at large $\Delta, \beta \simeq 1$ in the core so that here the omega effect is highly suppressed, and the fluid is approximately in solid-body motion. In the core $\alpha(\zeta)$ has a parabolic profile, and in fact $\alpha(\zeta) \simeq M \zeta^{2}$ to a good approximation. Thus the advection term $M \zeta^{2}-\alpha-\Omega$ in (3.21) is also strongly suppressed, another symptom of approximate solid-body rotation. As we move through the diffusive layers, $\alpha$ and $\beta$ adjust rapidly to the field-free outer solution where $\alpha=$ const., $\beta=0$.

It is not easy to gain an intuitive grasp of the field structure from the real and imaginary parts of $A$ and $B$, and so in figure 4 we reconstitute the field, showing 

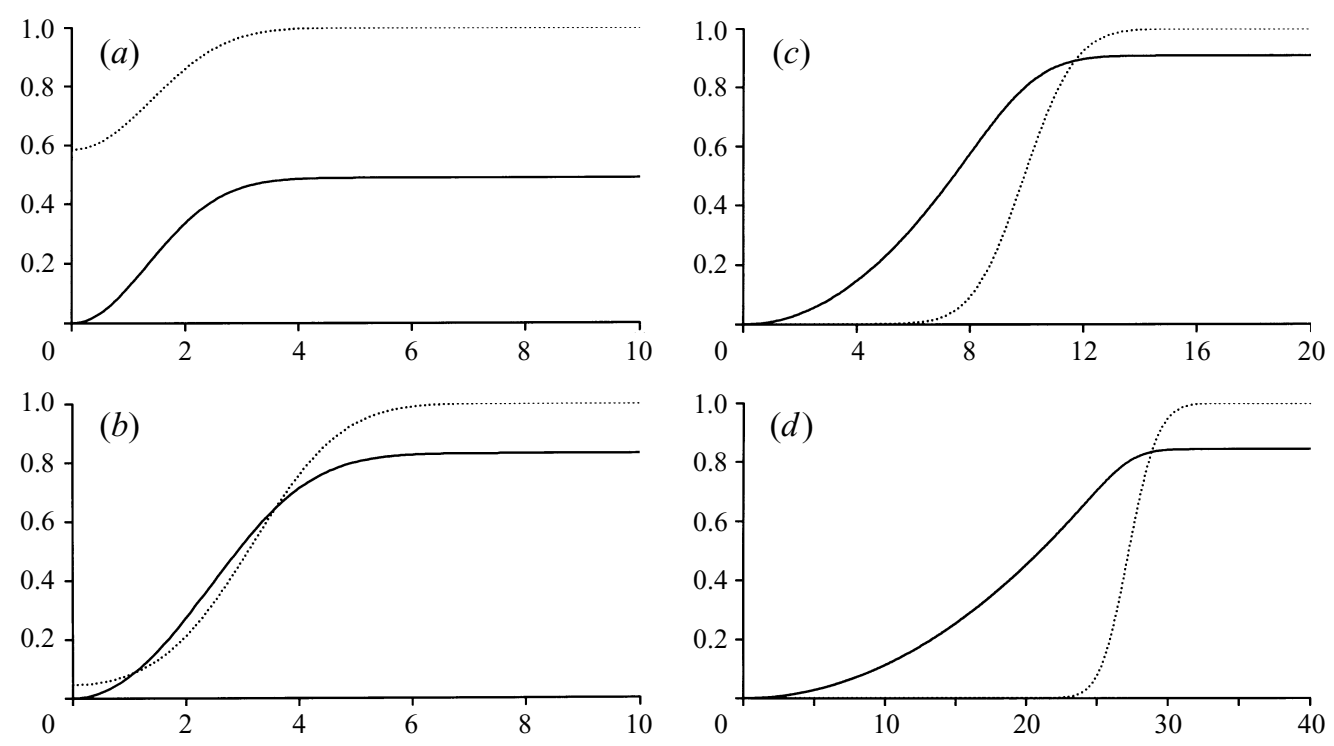

FIGURE 3. Nonlinear terms in equilibrated nonlinear states with $\sigma=1$. Shown are $\alpha(\zeta) / \Delta$ (solid) and $1-\beta(\zeta)$ (dotted), as functions of $\zeta$. In $(a-d) \Delta=0.5,1,2$ and 4 respectively.
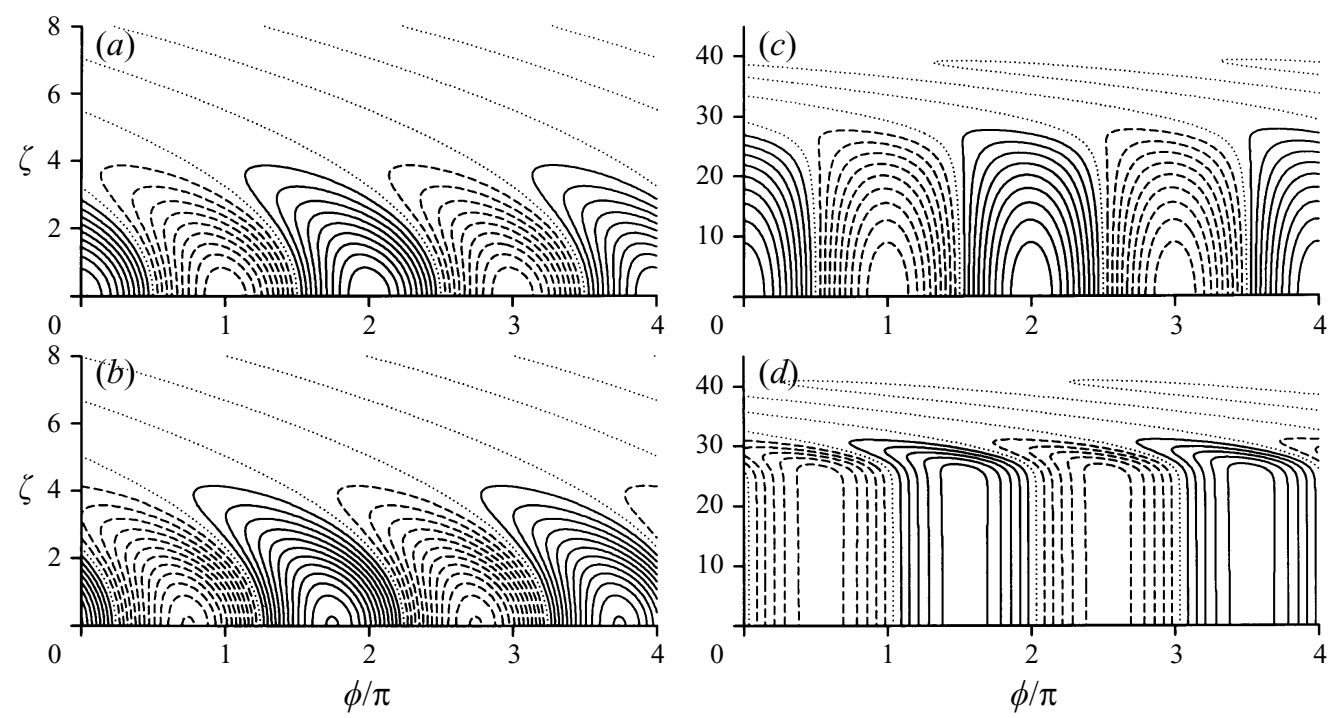

Figure 4. Contour plot of the radial and azimuthal fields at $z=0 . \operatorname{In}(a, b) \Delta=0.5$, while in $(c, d)$ $\Delta=4$. The radial field $A(\zeta) \exp (\mathrm{i} \phi)+$ c.c. is shown in $(a, c)$ and the azimuthal field $B(\zeta) \exp (\mathrm{i} \phi)+$ c.c. in $(b, d)$. Solid contours denote positive values and dashed contours negative ones: zero values are shown by dots. In $(a, b)$ the maximum radial field is unity and contour spacing is 0.1 ; in $(c, d)$ the maximum radial field is 8 and spacing is 0.8 in $(c), 0.4$ in $(d)$.

contour plots of the radial and azimuthal fields covering two periods in angle. In figure $4(a, b)$ for $\Delta=0.5$ the field resembles the linear eigenfunction with exponential decay as $\zeta \rightarrow \infty$. In figure $4(c, d)$, for $\Delta=4$, the breaking up of the domain into a core region and thin diffusive layers is apparent; in $(c)$ the radial component of field decays in a parabolic fashion out to roughly $\zeta=30$ and thereafter is practically zero, 


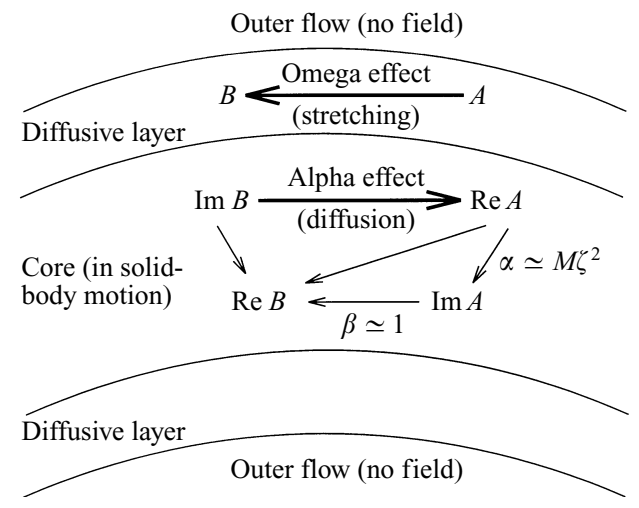

FIGURE 5. Schematic picture of the magnetic field structure and processes in the core region and thin diffusive bounding layers appropriate to the solution of (3.21), (5.1) when $\Delta \gg 1$.

while in $(d)$ the azimuthal field is constant as a function of $r$ in the core, but then is swept out in the diffusive layer and this rapid change of phase corresponds to the peak of $|\operatorname{Re} B|$ in this layer.

Recall that we are ultimately interested in the limit of small $M$, and so large $\Delta$, because we are seeking to reduce the true azimuthal wavenumber $m \equiv \varepsilon^{-1 / 3} M$ from $O\left(\varepsilon^{-1 / 3}\right)$ to smaller values and so follow the solution branch depicted in figure $1(b)$. In this limit the field appears to adopt an asymptotic structure, which we will obtain analytically. Although the numerical solution gives some information about the magnetic field in this limit, many significant questions can only be answered by analytical knowledge of the structure and scaling properties of the field. For example, we still do not know the physical mechanisms maintaining the field when the corelayer structure emerges for large $\Delta$, and we do not know whether this solution persists along the whole branch $1 \leqslant m \leqslant O\left(\varepsilon^{-1 / 3}\right)$ or whether there is a transition to different asymptotic scalings at some point. Finally we need information about the solution to enable us ultimately to justify the validity of the mean-flow system for large $R e$ (see $\S \S 2.3,6.2)$. With this background, in the following subsection we seek an analytical solution of (3.21) with $\Delta \gg 1$.

The results summarized in table 1 and figure 2 suggest the following behaviour for large $\Delta$. As $\Delta$ grows so $M=O\left(\Delta^{-2}\right)$ to a high degree of accuracy whilst $\operatorname{Re} B(0)$ appears to be very close to $(2 \Delta)^{-1}$. It is noteworthy that the frequency $\Omega$ reduces exceedingly rapidly; exactly how quickly is not easy to discern as the smaller $\Omega$ values may be dominated by numerical errors. It is also evident that the extent of the main activity zone increases with $\Delta$. An unambiguous definition for the limit of the core zone is not immediately apparent and, in its absence, we have chosen as a measure of the core width $\zeta_{0}$, defined to be the location at which the first zero of $\operatorname{Re} A$ occurs. Table 1 suggests that $\zeta_{0}$ rises slightly more quickly than $\Delta$. Finally, we are reminded by figure 3 that a very good approximation is $\alpha \simeq M \zeta^{2}$ and $\beta \simeq 1$ in the core.

\subsection{Analysis for $\Delta \gg 1$}

The full problem is to solve (3.21) subject to boundary conditions (5.1) in the $\Delta \gg 1$ limit and the motivation for our analysis is prompted largely by the numerical evidence described in $\$ 5.1$ above. For ease of discussion we divide our account into the core and diffusive layers; the general structure of the asymptotic solution and the mechanisms maintaining the field are shown schematically in figure 5. Some of the 
detail in this figure will become apparent later in this section. For the present note that the alpha effect, or conversion of azimuthal to radial field, occurs in the core region; here differential rotation and the omega effect are completely suppressed. The omega effect, the stretching of field by differential rotation, is 'expelled' from this core and occurs in the thin diffusive layers outside which the field decreases to zero. The omega effect represents the only energy source for the magnetic field to combat Ohmic dissipation, and so energy flows into the core from the diffusive layers.

The exact scalings that we use in terms of $\Delta$ are, in the main, far from clear at the outset but they can be established by forcing certain key balances to hold which necessitate that the core layer solutions should be brought smoothly to zero by the diffusive layer. However, rather than attempt to explain these balances now, we will first present the asymptotic analysis and then return to the question of the derivation of the salient scalings.

\subsubsection{The core region}

In the core it is convenient to decompose the fields and the operator $\mathscr{L}$ into real and imaginary parts according to

$$
A=A_{r}+\mathrm{i} A_{i}, \quad B=B_{r}+\mathrm{i} B_{i}, \quad \mathscr{L}=\mathscr{L}_{r}+\mathrm{i} \mathscr{L}_{i},
$$

with

$$
\mathscr{L}_{r}=\partial_{\zeta}^{2}-M^{2}\left(1+\sigma^{2}\right), \quad \mathscr{L}_{i}=M \zeta^{2}-\alpha-\Omega .
$$

The governing equations become

$$
\begin{aligned}
& \mathscr{L}_{r} A_{r}-\mathscr{L}_{i} A_{i}=-2 M B_{i}, \quad \mathscr{L}_{r} A_{i}+\mathscr{L}_{i} A_{r}=2 M B_{r}, \\
& \mathscr{L}_{r} B_{r}-\mathscr{L}_{i} B_{i}=(\beta-1) A_{r}, \quad \mathscr{L}_{r} B_{i}+\mathscr{L}_{i} B_{r}=(\beta-1) A_{i},
\end{aligned}
$$

with real quantities given by

$$
\alpha=2 \int_{0}^{\zeta}\left(A_{r} \partial_{\zeta} A_{i}-A_{i} \partial_{\zeta} A_{r}\right) \mathrm{d} \zeta, \quad \beta=2 A_{r} B_{r}+2 A_{i} B_{i} .
$$

For $\Delta \gg 1$ it turns out that the core extends over an $O\left(\Delta^{7 / 5}\right)$ range and so we define the core coordinate $X$ by

$$
\zeta=\Delta^{7 / 5} X
$$

All other quantities are expanded as series in powers of $\Delta^{-6 / 5}$. From the discussion in $\S 5.1$, we expand the scaled wavenumber with a leading $O\left(\Delta^{-2}\right)$ behaviour,

$$
M=\Delta^{-2}\left(M_{0}+\Delta^{-6 / 5} M_{1}+\cdots\right) .
$$

The fields are expanded as

$$
\begin{gathered}
A_{r}=\Delta\left(A_{r 0}+\Delta^{-6 / 5} A_{r 1}+\cdots\right), \quad A_{i}=\Delta^{-1 / 5}\left(A_{i 0}+\Delta^{-6 / 5} A_{i 1}+\cdots\right), \\
B_{r}=\Delta^{-1}\left(B_{r 0}+\Delta^{-6 / 5} B_{r 1}+\cdots\right), \quad B_{i}=\Delta^{1 / 5}\left(B_{i 0}+\Delta^{-6 / 5} B_{i 1}+\cdots\right),
\end{gathered}
$$

and the fact that the real and imaginary parts of $A$ and $B$ are treated differently is a consequence of our fixing the (arbitrary) phase of the field so that $A(0)=\Delta$ is real. These expansions then imply the forms for $\alpha, \Omega$ and $\beta$

$\alpha=\Delta^{4 / 5}\left(\alpha_{0}+\Delta^{-6 / 5} \alpha_{1}+\cdots\right), \quad \Omega=\Delta^{4 / 5}\left(\Omega_{0}+\Delta^{-6 / 5} \Omega_{1}+\cdots\right), \quad \beta=\beta_{0}+\Delta^{-6 / 5} \beta_{1}+\cdots$, 
and, finally, we then have

$$
\begin{gathered}
\mathscr{L}_{r}=\Delta^{-14 / 5} \partial_{X}^{2}-\Delta^{-4}\left(M_{0}^{2}+\Delta^{-6 / 5} 2 M_{0} M_{1}+\cdots\right)\left(1+\sigma^{2}\right), \\
\mathscr{L}_{i}=\Delta^{4 / 5}\left(M_{0} X^{2}-\alpha_{0}-\Omega_{0}\right)+\Delta^{-2 / 5}\left(M_{1} X^{2}-\alpha_{1}-\Omega_{1}\right)+\cdots .
\end{gathered}
$$

The expansions (5.5)-(5.8) are substituted into the governing equations (5.1), (5.4) and powers of $\Delta$ are equated; we do not write this out here. We take the core region to be defined as the zone in which $A_{r 0} \neq 0$, in accordance with the numerical results.

We begin with equation $(5.4 b)$ which at leading order $\Delta^{9 / 5}$ yields

$$
\left(M_{0} X^{2}-\alpha_{0}-\Omega_{0}\right) A_{r 0}=0 .
$$

In the core $A_{r 0} \neq 0$ and so the contents of the bracket vanish. Since $\alpha_{0}=0$ at $X=0$ we deduce that

$$
\alpha_{0}=M_{0} X^{2}, \quad \Omega_{0}=0 .
$$

Arguing similarly for $(5.4 b)$ at the following three orders yields

$$
\alpha_{1}=M_{1} X^{2}, \quad \alpha_{2}=M_{2} X^{2}, \quad \alpha_{3}=M_{3} X^{2}, \quad \Omega_{1}=\Omega_{2}=\Omega_{3}=0 .
$$

The differential advection term $M \zeta^{2}-\alpha-\Omega$ is zero in the core up to the orders considered, because in the expansion there is nothing to balance it. Only at the next order, $O\left(\Delta^{-3}\right)$, does the picture change, as we obtain new terms,

$$
\partial_{X}^{2} A_{i 0}+\left(M_{4} X^{2}-\alpha_{4}-\Omega_{4}\right) A_{r 0}=2 M_{0} B_{r 0} .
$$

We now look at equation (5.4c); given the vanishing of the advective terms (5.9), (5.10), there is nothing in the equation to balance the right-hand side at leading order, and the following three orders. Since $A_{r 0} \neq 0$ in the core we obtain

$$
\beta_{0}=1, \quad \beta_{1}=\beta_{2}=\beta_{3}=0 ;
$$

the stretching term $1-\beta$ is largely switched off in the core. At the following order, $O\left(\Delta^{-19 / 5}\right)$, we obtain

$$
\partial_{X}^{2} B_{r 0}-\left(M_{4} X^{2}-\alpha_{4}-\Omega_{4}\right) B_{i 0}=\beta_{4} A_{r 0} .
$$

Given the vanishing of advective and stretching terms (5.9), (5.10), (5.12), equation (5.4d) now yields at the first two non-trivial orders

$$
\begin{gathered}
\partial_{X}^{2} B_{i 0}=0, \\
\partial_{X}^{2} B_{i 1}-M_{0}^{2}\left(1+\sigma^{2}\right) B_{i 0}=0 .
\end{gathered}
$$

The solution of (5.14a) subject to the symmetry conditions of (5.1) is just

$$
B_{i 0}=C_{0}=\text { const. }
$$

Using (5.9), (5.10) again, equation (5.4a) yields

$$
\begin{gathered}
\partial_{X}^{2} A_{r 0}=-2 M_{0} B_{i 0}, \\
\partial_{X}^{2} A_{r 1}-M_{0}^{2}\left(1+\sigma^{2}\right) A_{r 0}=-2 M_{0} B_{i 1}-2 M_{1} B_{i 0} .
\end{gathered}
$$

These equations are important physically as they represent the conversion of $A$-field from $B$-field via the alpha effect (see figure 5). Once again, the symmetry constraints on $A_{r 0}$ lead to

$$
A_{r 0}=1-M_{0} C_{0} X^{2}
$$


We have so far obtained $A_{r 0}$ and $B_{i 0}$ explicitly; the remaining field components in the core are determined by our knowledge of $\alpha$ and $\beta$. The vanishing of differential rotation and stretching in the core determines these field components, as indicated in figure 5. From (5.4e), (5.9) we have

$$
\alpha_{0} \equiv 2 \int_{0}^{X}\left(A_{r 0} \partial_{X} A_{i 0}-A_{i 0} \partial_{X} A_{r 0}\right) \mathrm{d} X=M_{0} X^{2} .
$$

Differentiating this equation and substituting $A_{r 0}$ from (5.17) gives an ODE for $A_{i 0}$. The solution, applying (5.1), is simply

$$
A_{i 0}=M_{0} X^{2} / 2 .
$$

Finally, $(5.4 f),(5.12 a)$,

$$
\beta_{0} \equiv 2 A_{r 0} B_{r 0}+2 A_{i 0} B_{i 0}=1,
$$

fix $B_{r 0}$ in terms of the other field components, with

$$
B_{r 0}=1 / 2 \text {. }
$$

This completes our determination of the leading-order field in the core; we note that the forms of the core solutions, (5.15), (5.17), (5.19), (5.21), are entirely consistent with our earlier commentary on the numerical results in $\$ 5.1$. To summarize, the leading-order field structure in the core is

$$
\begin{gathered}
A=\Delta\left(1-M_{0} C_{0} X^{2}\right)+\Delta^{-1 / 5}\left(A_{r 1}+\frac{1}{2} \mathrm{i} M_{0} X^{2}\right)+\cdots, \\
B=\Delta^{1 / 5} C_{0}+\Delta^{-1}\left(\frac{1}{2}+\mathrm{i} B_{i 1}\right)+\cdots .
\end{gathered}
$$

Before moving on to the diffusive layer we make a few comments about pursuing the expansion to higher orders: this is straightforward provided the governing equations (5.4) are taken in the correct order, as done above. Here we merely note that the solutions of $(5.14 b)$ and $(5.16 b)$ are

$$
\begin{gathered}
A_{r 1}=\left[\frac{1}{2} M_{0}^{2}\left(1+\sigma^{2}\right)-M_{1} C_{0}-M_{0} C_{1}\right] X^{2}-\frac{1}{6} M_{0}^{3} C_{0}\left(1+\sigma^{2}\right) X^{4}, \\
B_{i 1}=C_{1}+\frac{1}{2} C_{0} M_{0}^{2}\left(1+\sigma^{2}\right) X^{2},
\end{gathered}
$$

where $C_{1}$ is some real constant which enters our discussion of the dynamics of the diffusion layer below.

Intriguingly, when the leading-order fields are substituted in (5.11) and then (5.13), we obtain

$$
\alpha_{4}=M_{4} X^{2}, \quad \Omega_{4}=0, \quad \beta_{4}=0 .
$$

Thus the extinction of differential rotation $M X^{2}-\alpha$, stretching $\beta-1$ and frequency $\Omega$ holds over at least five levels of the expansion. We have not tried to determine at what order these balances are finally broken.

\subsubsection{The diffusive layer}

The thin diffusive layer matches the core solution onto the outer region of zero field. Its location is given where our previous analysis breaks down, at the zero crossing of $A_{r 0}$. In terms of core variables, from (5.22), this is at $X_{0}$ with

$$
M_{0} C_{0} X_{0}^{2}=1 .
$$

In this layer, $A$ has size of order $\Delta^{-1 / 5}$ while $B$ is of size $\Delta^{1 / 5}$. To analyse this layer it 
is convenient to write $A$ as the core solution obtained above plus a modification $\Phi$ so that

$$
A=\Delta A_{r 0}+\Delta^{-1 / 5}\left(A_{r 1}+A_{i 0}\right)+\cdots+\Delta^{-1 / 5} \Phi_{0}+\Delta^{-7 / 5} \Phi_{1}+\cdots .
$$

We demand that $\Phi \rightarrow 0$ to the left of the diffusive layer (i.e. into the core), while the total radial field $A \rightarrow 0$ to the right, entering the field-free outer region. We also write

$$
B=\Delta^{1 / 5} \Psi_{0}+\Delta^{-1} \Psi_{1}+\cdots
$$

and require that $\Psi_{0}$ match onto $i C_{0}$ in the core (see (5.22)) and vanish into the outer zone.

Recall that for the core solution the key advective term $M \zeta^{2}-\alpha_{\text {core }}-\Omega$ is extremely small, in fact of $O\left(\Delta^{-26 / 5}\right)$, whereas $M \zeta^{2}$ and $\alpha_{\text {core }}$ individually are of size $O\left(\Delta^{4 / 5}\right)$. With the forms (5.26) above, it follows that in the diffusive layer $M \zeta^{2}-\alpha-\Omega=\widetilde{\alpha}+O\left(\Delta^{-26 / 5}\right)$, where $\widetilde{\alpha}$ is the modification to $\alpha_{\text {core }}$ that arises because of the new $\Phi$ terms in $A$ above. Now $\widetilde{\alpha}$ is of size $O\left(\Delta^{-2 / 5}\right)$, and can be calculated by substituting $(5.26 a)$ in $(3.21 d)$ and deleting terms not involving the $\Phi_{i}$. To have $\widetilde{\alpha}$ and $\partial_{\zeta}^{2}$ appear at leading order the layer must be of thickness $O\left(\Delta^{1 / 5}\right)$ in terms of $\zeta$. Thus we scale the layer on a variable $Y$ with

$$
\zeta=\Delta^{7 / 5} X_{0}+\Delta^{1 / 5} Y, \quad X=X_{0}+\Delta^{-6 / 5} Y .
$$

Within the diffusive layer, near the zero-crossing of $A_{r 0}$, the series (5.26a) can be written symbolically as

$$
A=\Delta^{-1 / 5} \widetilde{A}_{0}+\Delta^{-7 / 5} \widetilde{A}_{1}+\cdots
$$

(see (5.31a) below) and we can also write

$$
M \zeta^{2}-\alpha-\Omega=\Delta^{-2 / 5} \widetilde{\alpha}_{0}+\Delta^{-8 / 5} \widetilde{\alpha}_{1}+\cdots+O\left(\Delta^{-26 / 5}\right), \quad \beta=\widetilde{\beta}_{0}+\Delta^{-6 / 5} \widetilde{\beta}_{1}+\cdots .
$$

The leading-order equations for $A$ and $B$ become

$$
\left(\partial_{Y}^{2}-\mathrm{i} \tilde{\alpha}_{0}\right) \tilde{A}_{0}=0, \quad\left(\partial_{Y}^{2}-\mathrm{i} \tilde{\alpha}_{0}\right) \Psi_{0}=\left(\tilde{\beta}_{0}-1\right) \tilde{A}_{0},
$$

from (3.21), and include diffusion, the omega effect (see figure 5) and differential rotation; the alpha effect has dropped out here. The next-order equations for $A$ and $B$ are

$$
\left(\partial_{Y}^{2}-\mathrm{i} \tilde{\alpha}_{0}\right) \tilde{A}_{1}-\mathrm{i} \tilde{\alpha}_{1} \tilde{A}_{0}=2 \mathrm{i} M_{0} \Psi_{0}, \quad\left(\partial_{Y}^{2}-\mathrm{i} \tilde{\alpha}_{0}\right) \Psi_{1}-\mathrm{i} \tilde{\alpha}_{1} \Psi_{0}=\left(\tilde{\beta}_{0}-1\right) \tilde{A}_{1}+\beta_{1} \tilde{A}_{0} . \quad(5.30 a, b)
$$

In order to derive equations for the $\Phi_{i}$ and $\Psi_{i}$ we expand our core solutions (5.22), (5.23), for $A_{r 0}, A_{r 1}$ and $A_{i 0}$ in terms of the diffusion layer variable $Y$, to give field expansions within the diffusive layer which have the structure (cf. (5.26), (5.28))

$$
\begin{gathered}
A=\Delta^{-1 / 5}\left[-\lambda_{00} Y+\lambda_{01}+\mathrm{i} \lambda_{02}+\Phi_{0}\right]+O\left(\Delta^{-7 / 5}\right), \\
B=\Delta^{1 / 5} \Psi_{0}+O\left(\Delta^{-1}\right) .
\end{gathered}
$$

The real constants $\lambda_{i j}$ are defined by

$$
\lambda_{00}=2 M_{0} C_{0} X_{0}, \quad \lambda_{01}=\left[\frac{1}{3} M_{0}^{2}\left(1+\sigma^{2}\right)-M_{1} C_{0}-M_{0} C_{1}\right] X_{0}^{2}, \quad \lambda_{02}=\frac{1}{2} M_{0} X_{0}^{2} .
$$

Written in this way, the requirement that these solutions match with the core necessitates that $\Phi_{0} \rightarrow 0$ as $Y \rightarrow-\infty$ whilst as $Y \rightarrow \infty$ we require $\Phi_{0} \sim \lambda_{00} Y-\lambda_{01}-\mathrm{i} \lambda_{02}$ so that the total field vanishes.

The diffusive layer equations now follow from substitution into (5.29). The only term which requires some care is the integral which defines $\alpha(\zeta)$; we have already argued that the inclusion of the term $\Phi_{0}$ in $(5.31 a)$ means that within the diffusive zone 


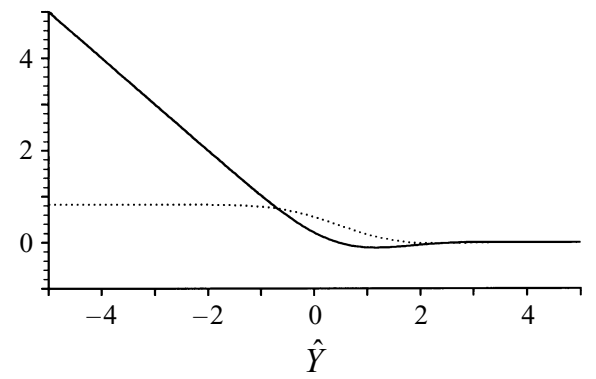

FIGURE 6. The real (solid line) and imaginary (dotted) parts of the total eigenfunction $-\hat{Y}+\mathrm{i} \hat{\lambda}_{02}+\hat{\Phi}_{0}$ in the diffusive layer.

$\alpha$ is perturbed by an $O\left(\Delta^{-2 / 5}\right)$ amount. The equation which results from (5.29a) for the leading-order adjustment $\Phi_{0}$ to the local expansion of the core $A$-field component is found to be invariant under a simple translation of the origin of the $Y$-coordinate. This is merely a reflection of the fact that the location of the diffusive layer cannot be uniquely determined on the $Y=O(1)$ lengthscale. It is also advantageous to scale the constants $\lambda_{i j}$ in (5.32) and unknowns in (5.31) according to

$$
\hat{\Phi}_{0}=\frac{\Phi_{0}}{\lambda_{00}^{1 / 2}}, \quad \hat{Y}=\lambda_{00}^{1 / 2}\left(Y-\frac{\lambda_{01}}{\lambda_{00}}\right), \quad \hat{\lambda}_{02}=\frac{\lambda_{02}}{\lambda_{00}^{1 / 2}},
$$

for then the determining equation for $\hat{\Phi}_{0}$ reduces to

$$
\frac{\mathrm{d}^{2} \hat{\Phi}_{0}}{\mathrm{~d} \hat{Y}^{2}}-\mathrm{i} \hat{\alpha}_{0}\left(-\hat{Y}+\mathrm{i} \hat{\lambda}_{02}+\hat{\Phi}_{0}\right)=0,
$$

with

$$
-\mathrm{i} \hat{\alpha}_{0}=\hat{Y}\left(\hat{\Phi}_{0}-\hat{\Phi}_{0}^{*}\right)+\mathrm{i} \hat{\lambda}_{02}\left(\hat{\Phi}_{0}+\hat{\Phi}_{0}^{*}\right)+\int_{-\infty}^{\hat{Y}}\left\{2\left(\hat{\Phi}_{0}^{*}-\hat{\Phi}_{0}\right)+\hat{\Phi}_{0} \frac{\mathrm{d} \hat{\Phi}_{0}^{*}}{\mathrm{~d} \hat{Y}}-\hat{\Phi}_{0}^{*} \frac{\mathrm{d} \hat{\Phi}_{0}}{\mathrm{~d} \hat{Y}}\right\} \mathrm{d} \hat{Y},
$$

and is to be solved subject to

$$
\begin{gathered}
\hat{\Phi}_{0} \rightarrow 0 \quad \text { as } \quad \hat{Y} \rightarrow-\infty, \\
\hat{\Phi}_{0} \sim \hat{Y}-\mathrm{i} \hat{\lambda}_{02} \quad \text { as } \quad \hat{Y} \rightarrow \infty,
\end{gathered}
$$

where $\hat{\lambda}_{02} \equiv M_{0} X_{0}^{5 / 2} / \sqrt{8}$.

This nonlinear integro-differential equation was solved using a code based on the same eigenvalue solver described briefly in connection with the strongly nonlinear system (3.21). Equation (5.34a) was cast as a system of first-order real equations and treated as an eigenproblem for $\hat{\lambda}_{02}$. It was found that the imposition of boundary conditions required a little care, especially that for $\hat{Y} \rightarrow-\infty$, otherwise the unwanted 'trivial solution' $\hat{\Phi}_{0}=\hat{Y}-\mathrm{i} \hat{\lambda}_{02}$ was identified. (Of course in practice the computation must be carried out on a finite $\hat{Y}$-interval and, if the naïve boundary condition $\hat{\Phi}_{0}=0$ is applied at the left-hand end of the interval, the routine inevitably settled on a solution of $(5.34 a)$ linear in $\hat{Y}$.) Eventually, in order to resolve this difficulty, we developed the asymptotic solution of $(5.34 a)$ as $\hat{Y} \rightarrow-\infty$ in order to determine a relationship between $\hat{\Phi}_{0}$ and $\mathrm{d} \hat{\Phi}_{0} / \mathrm{d} \hat{Y}$ in that limit. This, used as a boundary condition, was then enough to preclude the unwanted solution and enabled us to determine the required eigenfunction. In figure 6 we illustrate the real and imaginary parts of the 


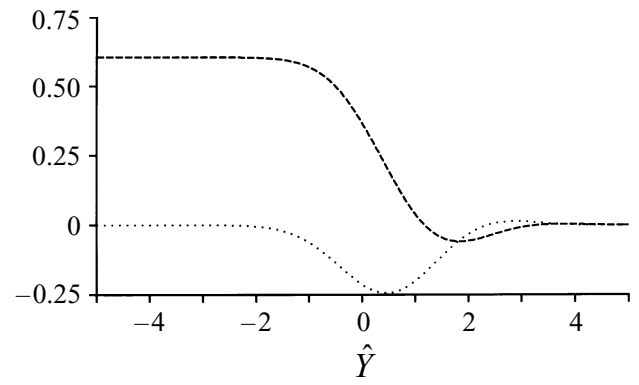

FIGURE 7. The real (wide dotted line) and imaginary (dashed) parts of the eigenfunction $\hat{\Psi}_{0}$ in the diffusive layer.

total eigenfunction $-\hat{Y}+\mathrm{i} \hat{\lambda}_{02}+\hat{\Phi}_{0}$ which shows how the $A$-component of the field is brought smoothly to zero from the core via the transition into the outer zone.

The leading-order $B$-component of the induced field in the diffusion layer is found by substituting $(5.31)$ in $(5.29 b)$. If we introduce the scaling

$$
\hat{\Psi}_{0}=\lambda_{00}^{1 / 2} \Psi_{0},
$$

and demand that this field component matches properly with the core solution $(5.22 b)$ as $\hat{Y} \rightarrow-\infty$ we are left with

$$
\frac{\mathrm{d}^{2} \hat{\Psi}_{0}}{\mathrm{~d} \hat{Y}^{2}}-\mathrm{i} \hat{\alpha}_{0} \hat{\Psi}_{0}=\left(\hat{\beta}_{0}-1\right)\left(-\hat{Y}+\mathrm{i} \hat{\lambda}_{02}+\hat{\Phi}_{0}\right)
$$

with $\hat{\alpha}_{0}$ given by $(5.34 b)$ and

$$
\hat{\beta}_{0}=\left(-\hat{Y}+\mathrm{i} \hat{\lambda}_{02}+\hat{\Phi}_{0}\right) \hat{\Psi}_{0}^{*}+\left(-\hat{Y}-\mathrm{i} \hat{\lambda}_{02}+\hat{\Phi}_{0}^{*}\right) \hat{\Psi}_{0} .
$$

This must be solved subject to

$$
\begin{aligned}
& \hat{\Psi}_{0} \rightarrow \mathrm{i} / 2 \hat{\lambda}_{02} \quad \text { as } \quad \hat{Y} \rightarrow-\infty, \\
& \hat{\Psi}_{0} \rightarrow 0 \quad \text { as } \quad \hat{Y} \rightarrow \infty .
\end{aligned}
$$

Given the numerical solution of (5.34) this linear problem for $\hat{\Psi}_{0}$ can be readily solved and the eigenfunction is reproduced in figure 7 . We notice in particular the manner in which $\operatorname{Re} \hat{\Psi}_{0} \rightarrow 0$ as $|\hat{Y}| \rightarrow \infty$ with a distinctive negative dip which is reassuringly reminiscent of the behaviour of $\operatorname{Re} B$ remarked upon in connection with the full numerical solutions of the strongly nonlinear system (3.21) and shown in figure 2 .

At this juncture we have determined the leading-order field components within the diffusive layer and have shown how they match with both the core solution outlined in \$5.2.1 and the zero-field outer zone. Unfortunately, we still have three important constants which we have been unable to isolate: $C_{0}$, which is the strength of the leading $B$-component on the symmetry line $\zeta=0$ (see (5.15)), the scaled wavenumber $M_{0}$, and the value $X_{0}$ which fixes the point at which the core solution gives way to the diffusive layer. These constants satisfy (5.25) and the numerical solution of the leading-order eigenproblem (5.34) determines

$$
\hat{\lambda}_{02} \equiv M_{0} X_{0}^{5 / 2} / \sqrt{8} \simeq 0.827 .
$$

The fact that the eigenproblems (5.34) and (5.36) for the leading-order eigenfunctions are invariant under a simple translation in $\hat{Y}$ and also under a scaling in $\lambda_{00}$ means 
that these solutions are insufficient to pin down $M_{0}, C_{0}$ and $X_{0}$ uniquely. In order to do this, it is strictly necessary to solve the eigenproblems (5.30) for the correction terms $\tilde{A}_{1}$ and $\Psi_{1}$ in the diffusive layer field expansions. It was found that in practice these boundary value problems are sufficiently complicated that accurate numerical solutions are difficult to achieve.

In preference to a full numerical attack on these higher-order equations, we note that for amplitude parameter $\Delta$ as small as 2, the solutions of the full system (3.21) as summarized in table 1 are very well approximated by the leading-order asymptotic forms postulated in this section. Indeed, $M \simeq 0.072 \Delta^{-2}+O\left(\Delta^{-16 / 5}\right)$ so that (5.25) and (5.37) lead to

$$
M_{0} \simeq 0.072, \quad C_{0} \simeq 0.857, \quad X_{0} \simeq 4.02 .
$$

These values yield asymptotic eigenfunctions which are entirely in agreement with the solutions of the full system (3.21) (compare the diffusive layer eigenfunctions shown in figures 6 and 7 with the forms of the eigensolutions of (3.21) near $\zeta_{0}$ when $\Delta=4$ as illustrated in figure $2 d$.) Therefore, although accurate solution of the second-order eigenproblems would be desirable for completeness, it appears that the effort needed to accomplish this would give us very little extra information beyond that already known. Moreover, we can be confident at this point that we have identified the appropriate large- $\Delta$ solution to within acceptable tolerance.

\subsection{Justification of the scalings}

Now that the coupled core-diffusive layer structure has been explained and used to deduce the large- $\triangle$ solution we can give a brief a posteriori argument for our choice of scalings. By definition of $\Delta$, we have $\operatorname{Re} A=O(\Delta)$ in the core zone and the numerical evidence of table 1 is strongly indicative that $M=O\left(\Delta^{-2}\right)$. Let us take $\operatorname{Im} A=O\left(\Delta^{a}\right)$ and $\zeta=\Delta^{b} X$ in the core, where $a$ and $b$ are numbers to be determined. From the core balance $\beta \simeq 1$ it is natural to scale $\operatorname{Re} B=O\left(\Delta^{-1}\right)$ and $\operatorname{Im} B=O\left(\Delta^{-a}\right)$. Finally, the balance between generation of $A$-field from $B$-field in equation (5.16a) leads to the relation $3+a=2 b$ between exponents.

We now move to consider the diffusive layer. Here we anticipate that both linear terms from $\operatorname{Re} A$ and constant terms from $\operatorname{Im} A$ in the core must play a role in the eigenproblem or else the relevant system is overspecified. This fixes the width of the diffusive layer at $X=O\left(\Delta^{a-1}\right)$, or $\zeta=O\left(\Delta^{a+b-1}\right)$. In this zone $A=O\left(\Delta^{a}\right)$ and, in order that the total field is brought to zero by the diffusive layer, the modification to the simple Taylor expansion of the $A$-field solution in the core must also be $O\left(\Delta^{a}\right)$. This induces a correction of $O\left(\Delta^{2 a}\right)$ in the nonlinear integral $\alpha$ (defined by $(3.21 d)$ ) and to have a diffusion-driven layer this correction must balance the second-order radial derivative in the definition of operator $\mathscr{L}$ (see (3.21c)). This necessitates $2 a+b=1$ which, in conjunction with the core restriction $3+a=2 b$, suggests that $a=-\frac{1}{5}$ and $b=\frac{7}{5}$. Given these sizes for quantities in the core and diffusive layers, the remaining details of the structure follow according to $§ 5.2$.

\section{Regimes of validity}

The purpose of this section is to tidy up a number of loose ends. First in $\S 6.1$ we verify that we can reduce $m$ to values of order unity, so that the strongly nonlinear analysis above does apply all the way down the branch indicated in figure $1(b)$. In $\S 6.2$ we pick up the discussion of $\$ 2.3$, and verify that the mean-flow system is valid for $R e \rightarrow \infty$ for fixed $R m \gg 1$, throughout the whole of this nonlinear branch. Finally 
in $\S 6.3$, we pin down precisely how big $R e$ must be compared to $R m$ for the nonlinear solutions in this paper to be valid. In all three sections we restrict consideration to the leading-order solution in both the core and in the diffusive layer.

\subsection{Extrapolation to $m=O(1)$}

Recall that we started in $\$ 4.2$ at the bifurcation with azimuthal wavenumber $m=$ $\varepsilon^{-1 / 3} M$, where $M=O(1)$ and $\varepsilon \ll 1$ is held fixed. We then moved into strongly nonlinear regimes, by introducing a new parameter $\Delta$ and setting $M \simeq \Delta^{-2} M_{0}$ with $M_{0}=O(1)$. This corresponds to reducing the true azimuthal wavenumber to $m \simeq \varepsilon^{-1 / 3} \Delta^{-2} M_{0}$. Now if it is legitimate to increase $\Delta$ up to values as large as $\Delta=\varepsilon^{-1 / 6}\left(M_{0} / m\right)^{1 / 2}$ with $m=O(1)$, then we have achieved our goal, to find nonlinear equilibrated states for the full range $1 \leqslant m \leqslant O\left(\varepsilon^{-1 / 3}\right)$ at high $R m$, as indicated in figure $1(b)$.

It turns out that this can be done. For $\Delta$ in the range $1 \ll \Delta \leqslant O\left(\varepsilon^{-1 / 6}\right)$, with core scalings it may be checked that the full system of equations (2.11), (2.15) for a single magnetic mode and mean flow continues to be approximated at leading order by the system (5.9), (5.12a), (5.14a), (5.16a) giving the leading-order core solution (5.22). Similarly with diffusive layer scalings, the full system continues to be approximated at leading order by (5.29), giving the structure obtained numerically. Furthermore the constants $M_{0}, X_{0}$ and $C_{0}$ continue to satisfy the relations (5.25), (5.37). (The manipulations required in order to establish all these results follow along the lines already explained and, in the interests of brevity, we do not give further details.)

In summary the structure obtained in $\$ 5$ appears to hold over the whole range $1 \leqslant m=o\left(\varepsilon^{-1 / 3}\right)$. When $m=O(1)$ and $\Delta=O\left(\varepsilon^{-1 / 6}\right)$ the appropriate scalings for the fields in the core, now of thickness $O\left(\varepsilon^{1 / 10}\right)$, are

$$
a=O\left(\delta^{1 / 2}\right), \quad b=O\left(\delta^{1 / 2} \varepsilon^{-1 / 5}\right) .
$$

The diffusive layer has thickness $O\left(\varepsilon^{3 / 10}\right)$ and inside the fields have magnitude

$$
a=O\left(\delta^{1 / 2} \varepsilon^{1 / 5}\right), \quad b=O\left(\delta^{1 / 2} \varepsilon^{-1 / 5}\right) .
$$

Thus the magnetic energy of the system is dominated by the $b$-field in the core and is of size

$$
E_{M}=O\left(\delta \varepsilon^{-3 / 10}\right)=O\left(R e^{-1} \mathrm{Rm}^{3 / 10}\right) .
$$

Note that for fixed $\operatorname{Pr}_{M}$ this scales as $R e^{-7 / 10}$ which at first sight appears unlikely, as it decreases with increasing $R e$, contrary to what one might expect in an experiment in which the velocity is increased from zero. However recall from (2.6) that we non-dimensionalized the magnetic field with respect to a velocity $U$. In dimensional variables, for a fixed experimental geometry and fluid ( $L, \eta$ and $v$ fixed), the magnetic energy in fact increases with $U$, as one might expect, as $E_{M}^{\operatorname{dim}}=O\left(U^{13 / 10}\right) \cdot \dagger$

Note that the layer containing the field always remains thin and so the outer spiral Couette flow is unchanged. This should be contrasted with the situation for Görtler vortices, which are spanwise periodic modes that may grow in suitably concave boundary layer flows. Linear and weakly nonlinear theory for short-wavelength Görtler vortices takes a mean-flow form very similar to that described in the present work (Hall 1982a,b) and, in particular, the modes occupy a thin region within the boundary layer. However, in a fully nonlinear state the amplitude of the vortex is sufficiently large that within the 'core' region of activity, which spreads itself over an $O(1)$ fraction of the boundary layer, the interaction of the fundamental component

$\dagger$ We are grateful to Dr A. Tilgner for pointing out this paradox and its resolution. 
of the vortex with itself drives a mean flow which is of comparable size to the undisturbed boundary layer. Then the presence of the vortex dramatically alters the detailed structure of the entire boundary layer (not just the active region but also the outer zones where the mode is exponentially small). The nonlinear structure can only be determined by numerical solution of a free boundary problem and details of this may be found in Hall \& Lakin (1988) or Hall (1990).

\subsection{Validity of the mean-flow system}

Now that we have a complete asymptotic picture of the saturated magnetic mode, we can check that at high $R e=1 / \delta$ (with $R m$ fixed) the fluctuating flow is $O(\delta)$ times the mean flow, as asserted in $\S 2.3$, and so the mean-flow system is valid. Recall that the equation for the fluctuating flow $\boldsymbol{u}^{\prime}$, linearized about the mean flow and neglecting viscosity, is (2.16). The equation is driven by the fluctuating tension force, of order $\delta$, and we require that it have a (non-singular) solution, $\boldsymbol{u}^{\prime}=O(\delta)$, both in the core and in the layer. In this equation we take the boundary conditions to be such that $\omega=O(1)$ in the region where the field is localized.

At the bifurcation the terms $\varpi+m \omega+k w$ are of size $O\left(\varepsilon^{1 / 3}\right)$. Subsequently, as the nonlinear solution branch is followed, these terms remain of $O\left(\varepsilon^{1 / 3}\right)$ in the diffusive layer and are even further suppressed (see (5.9), (5.10)) in the core as it emerges for large $\Delta$. Thus these terms may be neglected compared with the Coriolis terms involving $\omega$ in (2.16) throughout the solution branch.

For $\Delta \gg 1$, in the core the terms $r \partial_{r} \omega$ and $\partial_{r} w$ in (2.16) may also be neglected, since these are nonlinearly suppressed (see (5.12)). Thus when the core has formed, (2.16) reduces to (2.17), and as discussed in $\$ 2.3$, this is soluble for $\boldsymbol{u}^{\prime}$. In fact taking the curl, the solution can be written as

$$
-4 \mathrm{i} k \omega \boldsymbol{u}^{\prime}=\nabla \times \boldsymbol{F}^{\prime}
$$

and so $\boldsymbol{u}^{\prime}=O(\delta)$ (given $\omega=O(1)$ as assumed).

In the diffusive layer, we cannot neglect $r \partial_{r} \omega$ and $\partial_{r} w$ since these terms are $O(1)$ in the layer where the gradients increase from zero in the core to match the outer flow. Equations (2.16) then reduce to

$$
\begin{aligned}
-2 \omega v^{\prime} & =-\partial_{r} P^{\prime}+F_{r}^{\prime}, \\
2 \omega u^{\prime}+r u^{\prime} \partial_{r} \omega & =-2 \mathrm{i} m P^{\prime} / r+F_{\theta}^{\prime}, \\
u^{\prime} \partial_{r} w & =-2 \mathrm{i} k P^{\prime}+F_{z}^{\prime}, \\
\partial_{r} u^{\prime}+u^{\prime} / r+2 \mathrm{i} m v^{\prime} / r & +2 \mathrm{i} k w^{\prime}=0 .
\end{aligned}
$$

These scalings hold both for the diffusive layer and also at the bifurcation itself, before the coupled but distinct core-diffusive layer structure emerges. By taking the curl, we obtain the analogue of (6.4) as

$$
-4 \mathrm{i} k \omega \boldsymbol{u}^{\prime}+\left[\left(2 \mathrm{i} m r^{-1} \partial_{r} w-2 \mathrm{i} k r \partial_{r} \omega\right) u^{\prime},-\partial_{r}\left(u^{\prime} \partial_{r} w\right), r^{-1} \partial_{r}\left(r^{2} u^{\prime} \partial_{r} \omega\right)\right]=\nabla \times \boldsymbol{F}^{\prime},
$$

which can be used to determine $u^{\prime}$ from the radial component, and then $v^{\prime}$ and $w^{\prime}$. The only slight risk is that the quantity $-4 \mathrm{i} k \omega+2 \mathrm{i} m r^{-1} \partial_{r} w-2 \mathrm{i} k r \partial_{r} \omega$ might vanish at some point in the layer as the shears change from zero in the core to the free-stream values. In the absence of an analytical solution in the diffusive layer it is not obvious whether or not this can happen. However if a zero in this quantity did occur, it could easily be eliminated by modifying the boundary conditions (2.3) so as to add 
a constant to $\omega(r)$ (while keeping $\omega\left(r_{0}\right)$ non-zero and order one) $\dagger \uparrow$ Thus our results certainly hold for a wide range of boundary conditions, although we cannot specify them precisely a priori.

\subsection{Relative size of $R e$ and $R m$}

In our analysis we have taken the limit $R e \rightarrow \infty$ at fixed $R m$, to obtain the mean-flow system, and then taken $R m \rightarrow \infty$; we have checked above that this is legitimate. Clearly our results will hold for $R e$ much larger than some power of $R m$ : we now determine this power, by assessing the size of the fluctuating flow and its effect on the field. The conclusion is that the power is one, and so our results are correct provided $R e \gg R m$, for any amplitude $\Delta$ and so for any azimuthal wavenumber $m$. Thus our analysis is valid for $\operatorname{Pr}_{M} \ll 1$ and $R m, R e \gg 1$. The derivation is sketched below and we restrict attention to checking the validity only of the leading-order equations in $\S 5$.

We first estimate the fluctuating tension force $\boldsymbol{F}^{\prime} E^{2}$, where we write $E=\exp (\mathrm{im} \theta+$ $\mathrm{i} k z+\mathrm{i} \varpi t)$ for brevity. Now from (1.2), (2.12),

$$
\left(F_{r}^{\prime}, F_{\theta}^{\prime}, F_{z}^{\prime}\right)=\left(-a^{2}-b^{2}, a \partial_{r} b-b \partial_{r} a, a \partial_{r} c-c \partial_{r} a-a c / r\right)
$$

and so from the scalings (3.1), (3.15), (5.5), (5.6) we have in the core,

$$
\left(F_{r}^{\prime}, F_{\theta}^{\prime}, F_{z}^{\prime}\right) \sim \delta \varepsilon^{-1 / 3}\left(1+\mathrm{i} \Delta^{-6 / 5}\right)\left(\Delta^{2 / 5}, \mathrm{i} \Delta^{-1 / 5}, \mathrm{i} \Delta^{-1 / 5}\right) .
$$

(Note that $c$ scales like $b$.) Here we are using $\sim$ to denote order of magnitude, and we are keeping track of the size of real and imaginary parts individually with obvious notation; this is necessary to obtain the best estimate. From equations (2.17) for the fluctuating flow $\boldsymbol{u}^{\prime} E^{2}$ we find

$$
\left(u^{\prime}, v^{\prime}, w^{\prime}\right) \sim \delta \varepsilon^{-1 / 3}\left(1+\mathrm{i} \Delta^{-6 / 5}\right)\left(\mathrm{i} \Delta^{-1 / 5}, \Delta^{2 / 5}, \Delta^{2 / 5}\right) .
$$

We require this fluctuating flow to have a negligible effect in the induction equation. It couples $E^{n}$ modes in $\boldsymbol{b}$ to nearby modes $E^{n \pm 2}$ and, in particular, it couples the $E$ and $E^{-1}$ modes.

To see its effect we restrict to a magnetic field of the form $\boldsymbol{b}=(a, b, c) E+$ c.c. and the flow $\boldsymbol{u}=(0, r \omega, w)+\left[\left(u^{\prime}, v^{\prime}, w^{\prime}\right) E^{2}+\right.$ c.c. $]$; the induction equation $(2.8 a)$ for the $E$-mode becomes, using $\nabla \cdot \boldsymbol{b}=\boldsymbol{\nabla} \cdot \boldsymbol{u}=0$ to simplify some terms,

$$
\begin{gathered}
(\mathrm{i} \varpi+\mathrm{i} m \omega+\mathrm{i} k w) a=u^{\prime} \partial_{r} a^{*}+a^{*} \partial_{r} u^{\prime} / 2+3 a^{*} u^{\prime} / 2 r+\varepsilon\left(\nabla^{2} a-a / r^{2}-2 \mathrm{i} m b / r^{2}\right), \\
(\mathrm{i} \varpi+\mathrm{i} m \omega+\mathrm{i} k w) b+u^{\prime} r \partial_{r}\left(b^{*} / r\right)+\left(\partial_{r} u^{\prime}+u^{\prime} / r\right) b^{*} / 2 \\
=a r \partial_{r} \omega+a^{*} r \partial_{r}\left(v^{\prime} / r\right)+\left(\partial_{r} a^{*}+a^{*} / r\right) 2 v^{\prime}+\varepsilon\left(\nabla^{2} b-b / r^{2}+2 \mathrm{i} m a / r^{2}\right) .
\end{gathered}
$$

Now in the leading-order core analysis, equation $(6.10 a)$ is used at order $\delta^{1 / 2} \varepsilon^{1 / 2}$ $\left(\Delta^{-9 / 5}+\mathrm{i} \Delta^{9 / 5}\right)$ while equation $(6.10 b)$ is used at order $\delta^{1 / 2} \varepsilon^{1 / 6}\left(\Delta+\mathrm{i} \Delta^{-13 / 5}\right)$. Using the orders of magnitude (6.9) and core scalings in $\$ 5.2 .1$ it is straightforward to check that all the fluctuating terms in $(6.10 a, b)$ are negligible provided $\delta \ll \varepsilon$ (independently of $\Delta$, given that $1 \leqslant \Delta \leqslant O\left(\varepsilon^{-1 / 6}\right)$ ). While we rather restricted the magnetic and flow modes in writing down (6.10), it is clear that including more modes cannot change this result.

The calculation for the diffusive layer is similar but easier, since we need not treat real and imaginary parts separately. Instead of (6.8), (6.9) we have

$$
\left(F_{r}^{\prime}, F_{\theta}^{\prime}, F_{z}^{\prime}\right) \sim \delta \varepsilon^{-1 / 3}\left(\Delta^{2 / 5}, \Delta^{-1 / 5}, \Delta^{-1 / 5}\right),
$$

$\dagger$ In terms of the discussion in $\S 2.3$, this would correspond to increasing the local Ekman number so that the Coriolis terms dominate over those involving the shears $\partial_{r} \omega$ and $\partial_{r} w$. 


$$
\left(u^{\prime}, v^{\prime}, w^{\prime}\right) \sim \delta \varepsilon^{-1 / 3}\left(\Delta^{-1 / 5}, \Delta^{8 / 5}, \Delta^{8 / 5}\right)
$$

(both real and imaginary parts). In the leading-order layer analysis $(6.10 a)$ is used at order $\delta^{1 / 2} \varepsilon^{1 / 2} \Delta^{-3 / 5}$ and $(6.10 b)$ at order $\delta^{1 / 2} \varepsilon^{1 / 6} \Delta^{-1 / 5}$. Using (6.12) and the diffusive layer scalings in $\$ 5.2 .2$ it may then be verified that the fluctuating terms are again negligible when $\delta \ll \varepsilon$.

\section{Discussion}

We have analysed the structure and behaviour of equilibrated steadily rotating magnetic fields in a smooth Ponomarenko dynamo for $R e \gg R m \gg 1$ and all azimuthal wavenumbers $m$ in the range $1 \leqslant m \leqslant O\left(R m^{1 / 3}\right)$. We find that the equilibration mechanism is a strong suppression of the differential rotation or omega effect in the region where the field is localized. The alpha effect remains unchanged during saturation because it arises from the fixed, helical geometry.

The equilibrated field is concentrated in a cylindrical 'core' region which is in solid-body motion. The field essentially freezes the fluid flow here and does not move relative to the flow: magnetic reconnection processes largely cease, except for the alpha effect arising from the helical geometry. Bounding the core region are thin diffusive layers in which the omega effect, which is the energy source and a necessary ingredient of the dynamo, takes place. Magnetic energy is generated in these layers, and diffuses into the core region to maintain the frozen field against weak diffusion.

Smooth Ponomarenko dynamos are classified as kinematic 'slow' dynamos (e.g. Childress \& Gilbert 1995) because the linear growth rate tends to zero with increasing magnetic Reynolds number. There is no generally accepted definition of 'fast' and 'slow' for nonlinearly saturated dynamos. However, under any sensible definition the nonlinear states found here would be described as 'slow', since magnetic reconnection essentially ceases in the nonlinear regimes and the field is frozen, with weak diffusion and weak generation in balance (see Childress \& Gilbert 1995, chapter 12).

We have not studied the stability of the equilibrated states. It is possible that they could be unstable, and so not realizable in experiment or numerical simulation. Nevertheless the scalings and structure obtained in this paper would be relevant as describing unstable fixed points, with a significance for the dynamics in a more complicated system. For example, in view of results concerning 'inverse cascades' (Pouquet, Frisch \& Léorat 1976; Krause \& Meinel 1988; Gilbert \& Sulem 1990), one possibility is that in the full dynamics the states with $m>1$ could be unstable to pairing instabilities. At high $R m$ a seed field could approach an equilibrated state with a large value of $m$, which would then become unstable to the $m-1$ state, and then the $m-2$ state and so on down to $m=1$. While each state may be unstable, the field would spend a long period in its neighbourhood (see for example the studies of Krause \& Meinel 1988; Gilbert \& Sulem 1990). Numerical studies would be needed to decide what happens in the present system.

The key to our analysis is that at high Re the main feedback from the tension force is on the mean flow and so the field equilibrates at weak levels determined by viscosity. In particular the magnetic energy for one mode equilibrates at a level which is $O\left(\mathrm{Rm}^{3 / 10} / \mathrm{Re}\right)$. This is of course well below equipartition levels as the fluid energy is $O(1)$ and $R e \gg R m$. Because the magnetic field is viscously limited, our model is not very suitable for astrophysical or geophysical dynamos, where viscosity certainly is not a limiting factor for magnetic field amplitude. We have to recognize that the geometry of the Ponomarenko dynamo is too simple for the branch of solutions we 
follow (see figure $1 b$ ) to 'take off' and equilibrate at levels not limited by viscosity, the 'Malkus-Proctor scenario' (Malkus \& Proctor 1975), as is seen for example in geodynamo simulations. Of course there could be other branches of solutions in the Ponomarenko geometry with rather stronger fields, but we have not accessed these. It would be interesting to generalize the Ponomarenko geometry to allow the system to equilibrate at higher levels and to approach Taylor states. Two possibilities are to distort the streamsurfaces from cylinders, or to bend the flow into a torus; the framework for kinematic dynamo action in these cases may be found in Soward (1990).

Finally note that our analysis requires that the angular velocity at the radius of the cylindrical core does not vanish, $\omega\left(r_{0}\right) \neq 0$ (together with other conditions discussed in \$6.2), otherwise the mean-flow system is not valid since large fluctuating flows are excited. This condition is easily satisfied by appropriate choice of boundary conditions. However it does suggest that in an application, for example a dynamo experiment, the magnetic field would show most activity at radii where the angular velocity vanishes, if such exist; here the higher modes in the flow field would be excited and the picture would be very different, with probably the field equilibrating at a higher level.

We are grateful to Professor H. K. Moffatt, who originally suggested introducing a mean-flow feedback in the Ponomarenko dynamo (see Gilbert 1988b). We appreciate useful comments from Professor F. H. Busse, Professor C. A. Jones, Dr M. R. E. Proctor, Dr A. M. Rucklidge, Professor A. M. Soward, Dr A. Tilgner and the referees. A.D.G. gratefully acknowledges support from the Nuffield Foundation.

\section{REFERENCES}

BRAGINSKY, S. I. 1964a Self-excitation of a magnetic field during the motion of a highly conducting fluid. Zh. Exp. Teor. Fiz. SSSR 47, 1084-1098. (English transl.: Sov. Phys. JETP 20, 726-735 (1965).)

BRaginsky, S. I. 1964b Theory of the hydromagnetic dynamo. Zh. Exp. Teor. Fiz. SSSR 47, 2178 2193. (English transl.: Sov. Phys. JETP 20, 1462-1471 (1965).)

Brandenburg, A., Krause, F., Nordlund, A., Ruzmaikin, A. A., Stein, R. F. \& Tuominen, I. 1992 On the magnetic fluctuations produced by a large scale magnetic field. Unpublished.

Brandenburg, A., Nordlund, Å., Pulkkinen, P., Stein, R. F. \& Tuominen, I. 1990 3D simulation of turbulent cyclonic magnetoconvection. Astron. Astrophys. 232, 277-291.

Childress, S. \& Gilbert, A. D. 1995 Stretch, Twist, Fold: the Fast Dynamo. Lecture Notes in Physics: Monographs, vol. 37. Springer.

Dritschel, D. G. 1991 Generalized helical Beltrami flows in hydrodynamics and magnetohydrodynamics. J. Fluid Mech. 222, 525-541.

FeARn, D. R. 1994 Nonlinear planetary dynamos. In Lectures on Solar and Planetary Dynamos (ed. M. R. E. Proctor \& A. D. Gilbert), pp. 219-244. Cambridge University Press.

GaILitis, A. K. 1993 Experimental aspects of a laboratory scale liquid sodium dynamo model. In Solar and Planetary Dynamos (ed. M. R. E. Proctor, P. C. Matthews \& A. M. Rucklidge), pp. 91-98. Cambridge University Press.

Gailitis, A. K. \& Freiberg, YA. G. 1980 Non-uniform model of a helical dynamo. Magn. Gidrodin., no. 1, 15-19. (English transl.: Magnetohydrodynamics 16, 11-15.)

Gailitis, A. K., Karasev, B. G., Kirillov, I. R., Lielausis, O. A., Luzhanskij, S. M., OgorodNikov, A. P. \& PreslitskiJ, G. V. 1987 Liquid Metal MHD Dynamo Model. Physics Institute, Latvian SSR Academy of Sciences.

Gilbert, A. D. 1988 a Fast dynamo action in the Ponomarenko dynamo. Geophys. Astrophys. Fluid Dyn. 44, 241-258. 
Gilbert, A. D. $1988 b$ Topics in two-dimensional turbulence and dynamo theory. PhD Thesis, University of Cambridge.

Gilbert, A. D. \& Sulem. P.-L. 1990 On inverse cascades in alpha effect dynamos. Geophys. Astrophys. Fluid Dyn. 51, 243-261.

HaLl, P. $1982 a$ Taylor-Görtler vortices in fully developed or boundary layer flows: linear theory. J. Fluid Mech. 124, 475-494.

HaLl, P. $1982 b$ On the nonlinear evolution of Görtler vortices in non-parallel boundary layers. J. Inst. Maths Applics 29, 173-196.

HaLL, P. 1990 Görtler vortices in growing boundary layers: the leading edge receptivity problem, linear growth and the nonlinear breakdown stage. Mathematika 37, 151-189.

Hall, P. \& LaKin, W. D. 1988 The fully nonlinear development of Görtler vortices in growing boundary layers. Proc. R. Soc. Lond. A 415, 421-444.

Jones, C. A. \& RoberTs, P. H. 1990 Magnetoconvection in rapidly rotating Boussinesq and compressible fluids. Geophys. Astrophys. Fluid Dyn. 55, 263-308.

Krause, F. \& Meinel, R. 1988 Stability of simple nonlinear $\alpha^{2}$-dynamos. Geophys. Astrophys. Fluid Dyn. 43, 95-117.

Landman, M. J. 1990 Time-dependent helical waves in rotating pipe flow. J. Fluid Mech. 221, 289-310.

LÉorat, J. 1994 Linear dynamo simulations with time-dependent helical flows. Proc. 2nd Intl Conf. on Energy Transfer in MHD Flows (ed. A. Alemany \& P. Marty) 1994, Aussois, France. In: Magnetohydrodynamics 4, 367.

Lupian, E. A. \& Shukurov, A. M. 1992 Dynamo effect in realistic flows. Magn. Gidrodin. no. 3, $29-36$.

Malkus, W. V. R. \& Proctor, M. R. E. 1975 The macrodynamics of $\alpha$-effect dynamos in rotating fluids. J. Fluid Mech. 67, 417-444.

Moffatt, H. K. 1978 Magnetic Field Generation in Electrically Conducting Fluids. Cambridge University Press.

Ponomarenko, Yu. B. 1973 On the theory of hydromagnetic dynamos. Zh. Prikl. Mech. Tech. Fiz. (USSR) 6, 47-51.

Pouquet, A., Frisch, U. \& LÉORAT, J. 1976 Strong MHD turbulence and the nonlinear dynamo effect. J. Fluid Mech. 77, 321-354.

Roberts, P. H. \& Soward, A. M. 1992 Dynamo theory. Ann. Rev. Fluid Mech. 24, 459-512.

Ruzmaikin, A. A., Sokoloff, D. D. \& Shukurov, A. M. 1988 A hydromagnetic screw dynamo. J. Fluid Mech. 197, 39-56.

Ruzmaikin, A. A., Sokoloff, D. D., Solovyev, A. A. \& Shukurov, A. M. 1989 The CouettePoiseuille flow as a screw dynamo. Magn. Gidrodin. no. 1, 9-14.

Shukurov, A. M. \& Sokoloff, D. D. 1993 Evolution of magnetic fields in a swirling jet. In Solar and Planetary Dynamos (ed. M. R. E. Proctor, P. C. Matthews \& A. M. Rucklidge), pp. 271-274. Cambridge University Press.

Solovyev, A. A. 1985 axcitation of magnetic field by the axisymmetric motion of a conducting fluid. Izv. Akad. Nauk USSR, Fiz. Zemli, no. 4, 101-103.

Solovyev, A. A. $1985 b$ Description of the range of parameter values of the spiral Couette flow of a conducting fluid for which the excitation of magnetic field is possible. Izv. Akad. Nauk USSR, Fiz. Zemli, no. 12, 40-47.

Solovyev, A. A. 1985 c Existence of a magnetic dynamo for a dynamically admissible motion of a conducting fluid. Dokl. Akad. Nauk USSR 282, 44-48.

Solovyev, A. A. 1987 Magnetic field excitation by conducting fluid flow at high magnetic Reynolds numbers. Izv. Akad. Nauk USSR, Fiz. Zemli, no. 5, 77-80.

SowArD, A. M. 1990 A unified approach to a class of slow dynamos. Geophys. Astrophys. Fluid Dyn. 53, 81-107.

StUART, J. T. 1963 Hydrodynamic stability. In Laminar Boundary Layers (ed. L. Rosenhead), chapter IX. Oxford University Press.

TAYLOR, J. B. 1963 The magnetohydrodynamics of a rotating fluid and the Earth's dynamo problem. Proc. R. Soc. Lond. A 274, 274-283.

Vainshtein, S. I. \& Cattaneo, F. 1992 Nonlinear restrictions on dynamo action. Astrophys. J. 393, 199-203. 